\title{
Three-dimensional quasi-geostrophic vortex equilibria with $m$-fold symmetry
}

\author{
Jean N. Reinaud $\dagger$ \\ Mathematical Institute, University of St Andrews, North Haugh, St Andrews, KY16 9SS, UK
}

(Received xx; revised xx; accepted xx)

We investigate arrays of $m$ three-dimensional, unit Burger number, quasi-geostrophic vortices in mutual equilibrium whose centroids lie on a horizontal circular ring; or $m+1$ vortices where the additional vortex lies on the vertical 'central' axis passing through the centre of the array. We first analyse the linear stability of circular point vortex arrays. Three distinct categories of vortex arrays are considered. In the first category, the $m$ identical point vortices are equally spaced on a circular ring and no vortex is located on the vertical central axis. In the other two categories, a 'central' vortex is added. The latter two categories differ by the sign of the central vortex. We next turn our attention to finite volume vortices for the same three categories. The vortices consist in finite volumes of uniform potential vorticity and the equilibrium vortex arrays have an (imposed) $m$-fold symmetry. For simplicity all vortices have the same volume and the same potential vorticity, in absolute value. For such finite volume vortex arrays, we determine families of equilibria which are spanned by the ratio of a distance separating the vortices and the array centre to the vortices mean radius. We determine numerically the shape of the equilibria for $m=2$ up to $m=7$, for each three categories, and we address their linear stability. For the $m$-vortex circular arrays, all configurations with $m \geqslant 6$ are unstable. Point vortex arrays are linearly stable for $m<6$. Finite volume vortices may however be sensitive to instabilities deforming the vortices for $m<6$ if the ratio of the distance separating the vortices to their mean radius is smaller than a threshold depending on $m$. Adding a vortex on the central axis modifies the overall stability properties of the vortex arrays. For $m=2$, a central vortex tends to destabilise the vortex array unless the central vortex has opposite sign and is intense. For $m>2$, the unstable regime can be obtained if the strength of the central vortex is larger in magnitude than a threshold depending on the number of vortices. This is true whether the central vortex has the same sign as or the opposite sign to the peripheral vortices. A moderate strength like-signed central vortex tends however to stabilise the vortex array when located near the plane containing the array. On the contrary, most of the vortex arrays with an opposite-signed central vortex are unstable.

\section{Introduction}

Arrays of vortices in mutual equilibrium have arguably been of theoretical interest since the earliest works on vortex motion. Thomson (1883) first described the stability of $m$ two-dimensional identical point vortices equally spaced on a horizontal circular ring for $m=2$ up to $m=7$. Such a configuration is referred to as a circular $m$-vortex array. There is a very large body of literature dedicated to the study of vortex equilibria, in particular for two-dimensional vortices. Morikawa \& Swenson (1971) studied the effect of a central point vortex on the stability of an array of $m$ point vortices for two-dimensional vortices as

$\dagger$ Email address for correspondence: jean.reinaud@st-andrews.ac.uk 
well as for single-layer quasi-geostrophic shallow water vortices. The two-dimensional $m$ vortex problem was also revisited in depth by Kurakin \& Yudovich (2002). The stability of point vortex multipoles has also been to focus of numerous other studies including Aref (2009) in two dimensions and Kizner $(2011,2014)$ for a two-layer flow.

Thomson's seminal work also inspired further studies, including Dritschel (1985) where the point vortex configurations were generalised to arrays of two-dimensional finite area patches of uniform vorticity. Other configurations of finite area vortex equilibria with $m$-fold symmetries have been sought for two-dimensional vortices by Burbea (1982); Wu et al. (1984); Crowdy (2002, 2003); Kizner \& Khvoles (2004a,b); Xue et al. (2017) and for geophysical vortices by Kizner et al. (2007); Shteinbuch-Fridman et al. (2015); Kizner et al. (2017); Shteinbuch-Fridman et al. (2017); Reinaud et al. (2017) to name but a few studies.

Observations by the Juno spacecraft have recently revealed the presence of persistent polygonal arrays of cyclonic vortices on Jupiter's poles, see Adriani et al. (2018), motivating further studies of vortex equilibria in a three-dimensional, rapidly rotating and stratified environment. Large scale oceanic and atmospheric motions are also strongly influenced by the background planetary rotation and the background stable stratification of the fluid. The Quasi-Geostrophic (QG) model is the simplest dynamical model that takes these effects into account. It is asymptotically derived from the full equations of motion when both rotation and stratification effects dominate the flow evolution. In this framework, the flow can be fully described by the (slow) evolution of a materially conserved scalar quantity: the potential vorticity, see Vallis (2006). Vortices abound in the oceans and the atmosphere, see Ebbesmeyer et al. (1986); Chelton et al. (2011); Peterson et al. (2013); Zhang et al. (2014) and many other studies. These vortices can be defined as contiguous regions of potential vorticity.

The main objective of the present paper is to study, for the first time, equilibria for $m(m \geqslant 2)$ three-dimensional, unit Burger number, finite volume vortices of uniform potential vorticity located on a circular ring, within the QG approximation, in a rapidly rotating, continuously stratified fluid.We also examine the effect of the addition of a vortex on the vertical axis passing through the centre of the ring, a problem often referred in the literature as the $m+1$-vortex problem, see Sokolovskiy \& Verron (2008). Pairs of three-dimensional, continuously stratified quasi-geostrophic co-rotating vortices in mutual equilibrium were first analysed in Reinaud \& Dritschel (2002) while pairs to counter-rotating vortices were discussed in Reinaud \& Dritschel (2009). Additionally, a special class of three-vortex equilibria is discussed by Reinaud \& Carton (2015). Configurations of three-dimensional QG vortices arranged in a nearly regular pattern can be the result of the destabilisation of a torus of potential vorticity as shown by Reinaud \& Dritschel (2018b).

In this paper we show that unstable finite volume vortex equilibria can be found in some part of the parameter space for all the values of $m$ considered. For the $m$-vortex problem however, point vortices arrays are linearly stable for $m<6$. Nevertheless, finite volume vortices can be sensitive to modes of instability deforming the vortices for $m<6$ if the vortices are close enough to each other. For $m=2$, the addition of a central like-signed vortex destabilises the system. For $m>2$, adding a weak central like-signed vortex tends to stabilise the vortex array, in particular if the central additional vortex is located at a small enough height from the other vortices. An intense like-signed central vortex may however induce instability. The $2+1$-vortex system with an opposite-signed central vortex is also unstable unless the central vortex is intense. For $m>3$, adding an opposite-signed vortex on the central axis may destabilise the vortex arrays, and linearly stable solutions are found in fewer, if any, parts of the parameter space. 
The paper is organised as follows. Section 2 describes the mathematical model and the numerical tools used in the study. The main results for point vortices are discussed in $\S 3$ while the results for finite volume vortices are presented in $\S 4$. Conclusions are presented in $\S 5$.

\section{Mathematical setup}

We consider an adiabatic, inviscid, three-dimensional, horizontally and vertically unbounded, continuously stratified, rapidly rotating fluid. For simplicity we assume that the background rotation is uniform so that the Coriolis frequency $f$ is constant. We also assume that the buoyancy frequency $N$, defined by $N^{2}=g \rho_{0}^{-1} \mathrm{~d} \rho / \mathrm{d} z$ under the Boussinesq approximation, is constant so that the stratification is linear with depth. Here $g$ is the gravitational acceleration, $\rho_{0}$ is the mean density and $\rho(z)$ is the basic state density. The Boussinesq approximation assumes that the density variations are small compared to the mean density. This assumption is valid for the oceans. For convenience we rescale the physical vertical coordinate by the ratio $N / f$. Typically $N / f \gg 1$ in most parts of the oceans, see Dijkstra (2008). In this vertically stretched reference frame the equations become independent of $N$ and $f$, hence our results are valid for all values of $N / f$. We define the Froude number $F r=U /(N H)$, where $U$ is a characteristic scale of horizontal velocity and $H$ is a characteristic vertical length scale, and the Rossby number $R o=U /(f L)$, where $L$ is a characteristic horizontal length scale. For rapid background rotation $R o \ll 1$ and strong stratification $F r^{2} \ll R o$, the Boussinesq equations can be asymptotically expanded in terms of small $R o$ and $F r$ to obtain the quasi-geostrophic (QG) model (see Vallis, 2006 for details). The equations read

$$
q=\frac{\partial^{2} \psi}{\partial x^{2}}+\frac{\partial^{2} \psi}{\partial y^{2}}+\frac{\partial^{2} \psi}{\partial z^{2}}
$$

with

$$
\frac{\mathrm{D} q}{\mathrm{D} t}=\frac{\partial q}{\partial t}-\frac{\partial \psi}{\partial y} \frac{\partial q}{\partial x}+\frac{\partial \psi}{\partial x} \frac{\partial q}{\partial y}=0
$$

where $q$ defined by equation (2.1) is the QG potential vorticity anomaly, hereinafter referred to as $\mathrm{PV}$ for simplicity, $\psi$ is the streamfunction and $\mathrm{D} / \mathrm{D} t$ stands for the material derivative. Equation (2.2) states that PV is materially conserved for an adiabatic, inviscid fluid. It should be noted that the full dynamics is controlled by the PV, $q$, a single scalar quantity. Equation (2.1) can formally be inverted using the Green's function for the three-dimensional Laplacian

$$
G\left(\boldsymbol{x} ; \boldsymbol{x}^{\prime}\right)=-\frac{1}{4 \pi\left|\boldsymbol{x}-\boldsymbol{x}^{\prime}\right|},
$$

which by construction provides the streamfunction, evaluated at $\boldsymbol{x}=(x, y, z)$, induced by a point vortex of unit intensity located at $\boldsymbol{x}^{\prime}=\left(x^{\prime}, y^{\prime}, z^{\prime}\right)$. Differentiating explicitly the Green's function with respect to $x$ and $y$ respectively gives the velocity $v=\partial \psi / \partial x$ and $u=-\partial \psi / \partial y$ induced by the point vortex. Second order derivatives provide the velocity gradients which are used to study the linear stability of the point vortex arrays, see appendix A for details. For finite core vortices, the inversion of equation (2.1) leads to volume integrals. The fluid domain is represented by horizontal layers of equal thickness $\Delta z$ in which the Green's function (and its derivatives) can be integrated analytically. The remaining (horizontal) surface integrals are converted to contour integrals over the 

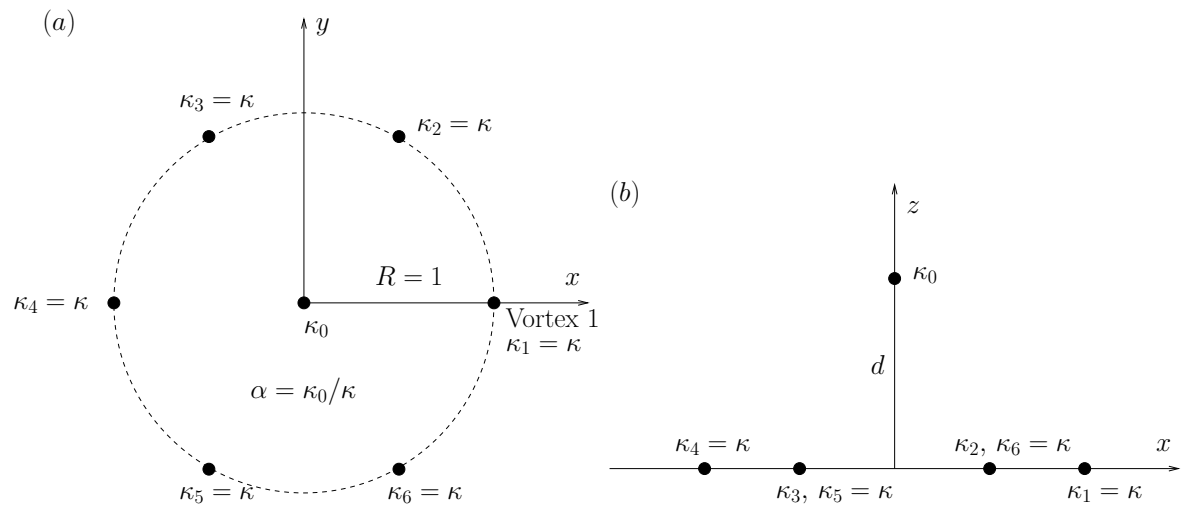

Figure 1. Geometry of the point vortex array and definition of the parameters presented for $6+1$ vortices: $(a)$ view from the top in the $x, y$-plane, $(b)$ side view in the $x, z$-plane.

contours bounding the horizontal cross-sections of the uniform PV vortices using Green's theorem.

The numerical method used to perform the simulation of the evolution of the flow is the purely Lagrangian Contour Surgery algorithm introduced by Dritschel (1988) for two dimensional flows and adapted to three-dimensional QG flows by Dritschel \& Saravanan (1994); Dritschel (2002). Contour Surgery is an extension on Contour Dynamics (see Zabusky, Hugues \& Roberts, 1979) which allows one to control the complexity of the vortex bounding contours by topological reconnections.

To obtain finite volume vortex equilibria, we use an iterative method which makes the vortex bounding contours converge to streamlines. The approach is based on a method developed by Pierrehumbert (1980) for two-dimensional flows and adapted to three-dimensional QG flows by Reinaud \& Dritschel (2002) and further used in Reinaud \& Dritschel (2009); Reinaud \& Carton (2015). The method is presented in Appendix B. The linear stability of the finite volume vortex arrays is addressed by analysing deformation modes of the vortex bounding contours (Reinaud \& Dritschel, 2002) and is briefly described in Appendix C. It includes a mode representing the displacement of the full contours, hence the relative displacement of the vortices.

\section{Point vortices}

We first consider arrays of point vortices. We refer to the vortices lying along the ring as the peripheral vortices. Vortex $i$ carries an intensity or 'charge' of potential vorticity $\Gamma_{i}$ which has the physical dimension of a volume integrated PV, see for example Gryanik (1983). The $m$ peripheral vortices are identified by their index $1 \leqslant i \leqslant m$. If an additional central vortex is present, it is identified by the index 0 . For convenience, we define the strength of vortex $i$ by $\kappa_{i}=\Gamma_{i} /(4 \pi)$. The point vortex problem has a unique length scale and a unique time scale which can be both chosen arbitrarily. The length scale is set by the radius $R=1$ of the ring on which the $m$ peripheral vortices are located, and the time scale is implicitly defined by taking $\kappa_{i}=\kappa=1$ for $1 \leqslant i \leqslant m$, where $\kappa$ is the common strength of the peripheral vortices. Without loss of generality, the ring of vortices is located on the horizontal plane $z=0$. The location of the $m$ peripheral vortices at $t=0$ is $\left(x_{i}, y_{i}, z_{i}\right)=\left(\cos \theta_{i}, \sin \theta_{i}, 0\right)$ where $\theta_{i}=2 \pi(i-1) / m$. The central vortex has strength $\kappa_{0}$ and is located at $(0,0, d)$. We take $d \geqslant 0$ without loss of generality. We define $\alpha=\kappa_{0} / \kappa$. The general geometry of the vortex array is shown in figure 1 . 
(a)

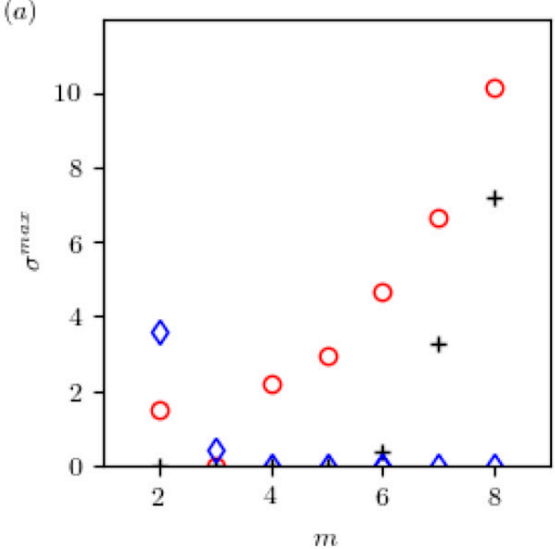

(b)

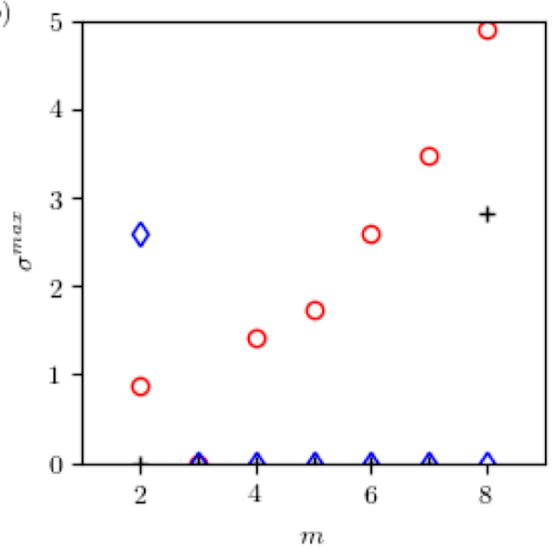

FIGURE 2. (a) Maximum growth rate $\sigma^{\max }$ versus the number of vortices $m$ for QG point vortices in a three-dimensional, continuously stratified fluid. The results for the $m$ vortex problem are indicated by black + , and the $m+1$ vortex problem with $d=0$ and $\alpha=1$ by blue $\diamond$ while $\alpha=-1$ by red $\circ$. (b) Same but for two-dimensional vortices for comparison.

We analyse the linear stability of the $m$-vortex arrays and of the $m+1$-vortex arrays for $d=0$ and $\alpha= \pm 1$ for $2 \leqslant m \leqslant 8$. When $d=0$, all $m+1$ vortices are co-planar. We analyse the normal modes of perturbation of the horizontal locations of the vortices $\left(x_{i}^{\prime}, y_{i}^{\prime}, 0\right)$. We do not consider perturbations of the vertical location of the vortices which could also modify the distance separating the vortices as no external quasi-geostrophic flow may move the point vortices in the vertical direction. We do not consider perturbations on the strength of the vortices neither. These modes of pertubations have a time dependence proportional to $\mathrm{e}^{\sigma t}=\mathrm{e}^{\sigma_{r} t}\left(\cos \sigma_{i} t+\mathrm{i} \sin \sigma_{i} t\right)$, where the real part of $\sigma$ is the growth rate of the mode, $\sigma_{r}$, and its imaginary part, $\sigma_{i}$, is its frequency. There are $n_{m}=2 \mathrm{~m}$ modes for the $m$-vortex problem or $n_{m}=2 m+2$ modes for the $m+1$-vortex problem. This approach is the same as the one used in different contexts in Reinaud \& Carton (2015, 2016) and is further described in Appendix A.

Figure 2 shows the maximum growth rate $\sigma^{\max }=\max _{1 \leqslant j \leqslant n_{m}}\left\{\sigma_{r_{j}}\right\}$ versus the number of peripheral vortices $m$. For comparison, similar results for two-dimensional vortices are included. The results, presented here, are obtained using the same numerical technique as for the QG computations but adapted to the two-dimensional situation. For the twodimensional vortices, the circulation of the peripheral vortices is set to $\Gamma_{2 D}=2 \pi$. When a central vortex is added, $d=0$ by construction, and again we use a central vortex of circulation $\Gamma_{0}= \pm \Gamma_{2 D}= \pm 2 \pi$. The parameter $\alpha$ is also used to denote the circulation ratio of the central vortex to the peripheral ones.

For the $m$-vortex problem, results shown in figure 2 indicate that the three-dimensional QG vortex array is 'less linearly stable' than the equivalent two-dimensional one. Indeed the two-dimensional vortex arrays are linearly stable for $m \leqslant 7$, see figure $2(b)$ and Thomson (1883); Kurakin \& Yudovich (2002), whereas the three-dimensional QG vortex arrays are only linearly stable for $m \leqslant 5$, see figure $2(a)$. Recall that the difference between the two situations lies in the nature of the Green's function which is $G_{2 D}\left(\boldsymbol{x}^{\prime} ; \boldsymbol{x}^{\prime}\right)=(1 / 2 \pi) \ln \left|\boldsymbol{x}-\boldsymbol{x}^{\prime}\right|$ for the two-dimensional case in contrast with (2.3). Hence the velocity gradients are proportional to $r^{-3}$ in QG, where $r=\left|\boldsymbol{x}-\boldsymbol{x}^{\prime}\right|$ is the distance between the source and the evaluation point, compared to $r^{-2}$ for the two dimensional vortices. 

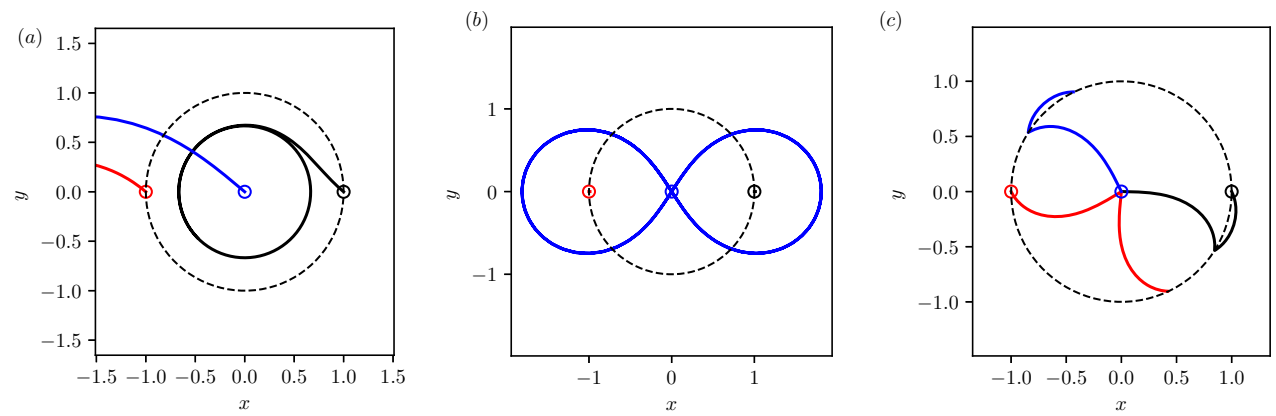

Figure 3. Trajectories of the point vortices for the unstable $2+1$-vortex problem for $(a)$ $\gamma=-1,(b) \gamma=0$, and $(c) \gamma=1$.

We also observe a strong influence of the presence of a central vortex. For $d=0$, $\alpha=1$ and $m>3$ the presence of the like-signed central vortex stabilises the array. The vortex array indeed remains linearly stable for $m=6$ up to 8 . The vortex array with $m=9$ is linearly unstable. Similar stabilising effects of a central, like-signed vortex, were first observed for two-dimensional geostrophic vortices by Morikawa \& Swenson (1971). It should be noted that the like-signed central vortex has however a destabilising effect for a small number of peripheral vortices $m=2,3$. On the other hand, the presence of a co-planar opposite-signed central vortex with $\alpha=-1$ destabilises the ring, except for the special case $m=3$. Overall, these trends are also observed for the two-dimensional case, as shown in figure $2(b)$.

We next show the nonlinear evolution of a selection of point vortex arrays. Figure 3 shows the trajectories of the point vortices for $2+1$-vortex arrays with $\alpha=-1,0$, and 1 . All trajectories are shown in the reference frame steadily rotating with the equilibrium. In this reference frame, the departure of the vortices from their initial position is the result of instability. In the case where $\alpha=-1$, shown in figure 3(a), the central vortex and each peripheral vortices have equal and opposite strength. The central and one of the peripheral vortices get closer together to form a vortex dipole which moves away from the origin. This vortex dipole has an overall zero strength as the strengths of the vortices compensate. To conserve the angular impulse $J=2 \pi \sum_{i=0}^{2} \kappa_{i}\left(x_{i}^{2}+y_{i}^{2}\right)=2 \pi \kappa R^{2}$, the other peripheral vortex orbits around the origin. For $\alpha=0$, the central vortex has zero strength and thus is a passive particle. The remaining two vortices are stable, see figure $2(a)$. The evolution of the vortex array is shown in figure $3(b)$. The two peripheral vortices, shown in red and black, remain indeed at their initial location. The passive particle however moves away from its initial location which is an hyperbolic critical point. It should be noted that instabilities have also be found in shallow water for such degenerate tripoles, in which the central pole is passive, by Kizner (2014), see in particular their figure 5 . This is further detailed below when we address the influence of the parameter $\alpha$.

For $\alpha=1$, the vortex array is unstable. The evolution of the vortices is shown in figure $3(c)$. Here all three vortices have equal strength. The trajectories indicate that one of the peripheral vortices may move towards the origin while the central vortex moves outward to conserve both linear and angular impulses. Similar behaviours where the central vortex moves towards the ring while one of the peripheral vortices moves towards the centre have been observed for larger values of $m$ as shown below.

Results presented in figure 2(a) also indicate that the $3+1$-vortex array with $\alpha=1$ is unstable. The evolution of the vortices is shown in figure 4 . The trajectory of the vortices appear to be chaotic. We also see that all vortices transitorily pass near the origin. For a 


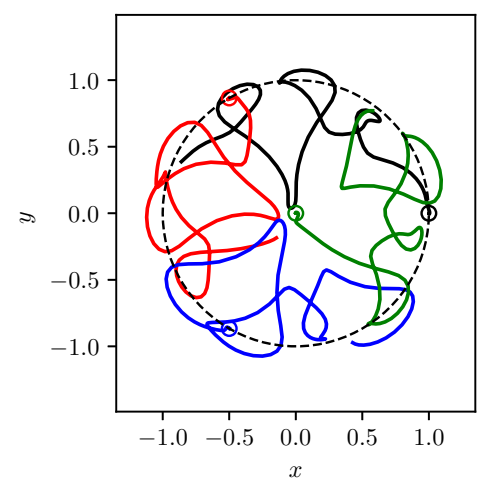

Figure 4. Trajectories of the point vortices for the unstable $3+1$-vortex problem for $\gamma=1$.
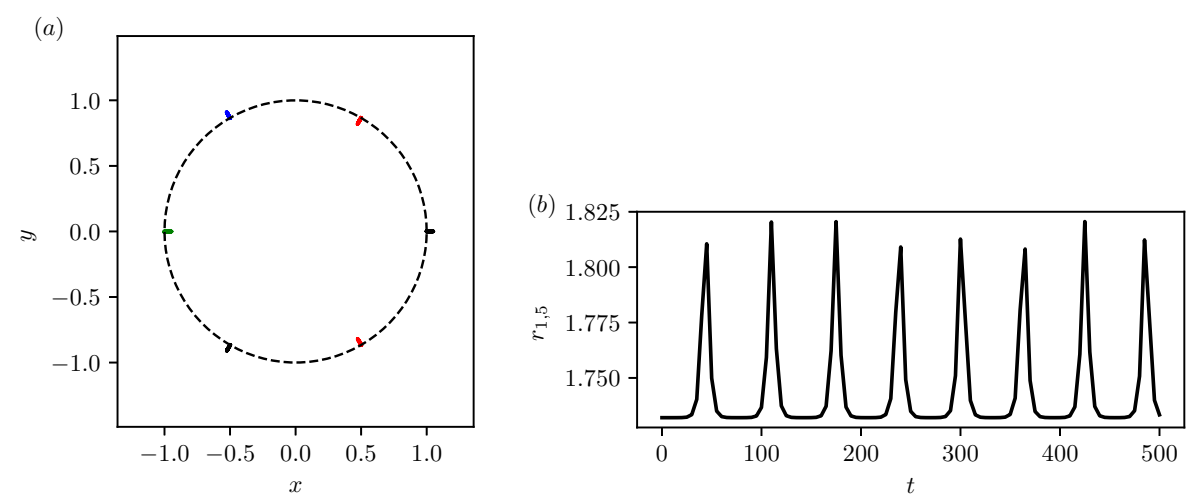

Figure 5. (a) Trajectories of the point vortices for the unstable 6 -vortex problem for $0 \leqslant t \leqslant 500$ (left). The unfilled circle indicated the initial position of the vortices. The dashed circle indicate the ring of radius $R=1$ where the vortices initially lie. (b) distance $r_{1,5}=\left|\boldsymbol{x}_{1}-\boldsymbol{x}_{5}\right|$ between the vortex 1 and vortex 5 vs time (right).

larger number of vortices $(m>4)$ and $\alpha=1$, the results (not shown) also indicate that the unstable equilibria lead to a chaotic motion of the vortices.

The evolution of the weakly unstable 6 -vortex array is shown in figure 5 . The weak instability results in a very small oscillatory motion of the vortices. The oscillation is due to nonlinear effects. The oscillation is better seen by plotting on the evolution of the distance $r_{1,5}=\left|\boldsymbol{x}_{1}-\boldsymbol{x}_{5}\right|$ between vortex 1 and vortex 5 , initially located at $(1,0,0)$ and $(-1 / 2,-\sqrt{3} / 2)$ respectively. After an exponential growth of the distance $r_{1,5}$ from its initial value, nonlinear effects bring the vortices back to their initial positions. This relative motion repeats quasi periodically. The evolution of the unstable 7 -vortex array is shown in figure 6 . In this case the instability is much stronger and the point vortices have an apparent chaotic motion. It can be noted that one of the 7 peripheral vortices moves (temporarily) near the centre of the array indicating that the stable $6+1$-vortex array acts as an attractor.

By symmetry, the $m+1$-vortex array remains in steady rotation even if $d \neq 0$ and $|\alpha| \neq 1$, provided the central vortex is located on the vertical axis passing through the centre of the ring, which is the rotation axis of the system. Moreover, in this case the system remains in steady rotation for all values of $\alpha$. The central vortex strength $\kappa_{0}$ and its vertical location $d$ modifies the angular velocity of the equilibrium but does not break 


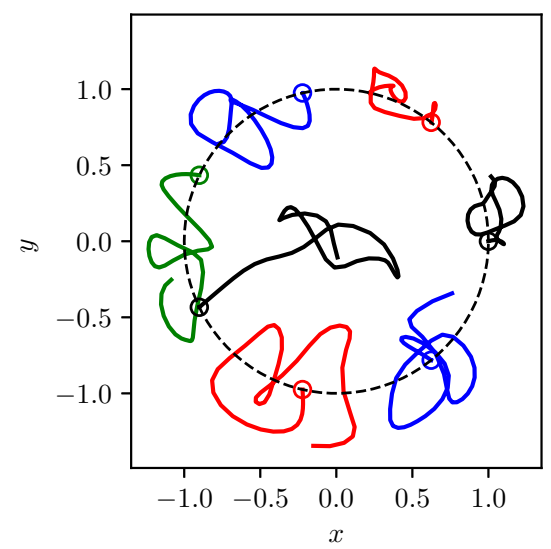

Figure 6. (a) Trajectories of the point vortices for the unstable 7 -vortex problem for $0 \leqslant t \leqslant 10$. The unfilled circle indicated the initial position of the vortices.

(a)

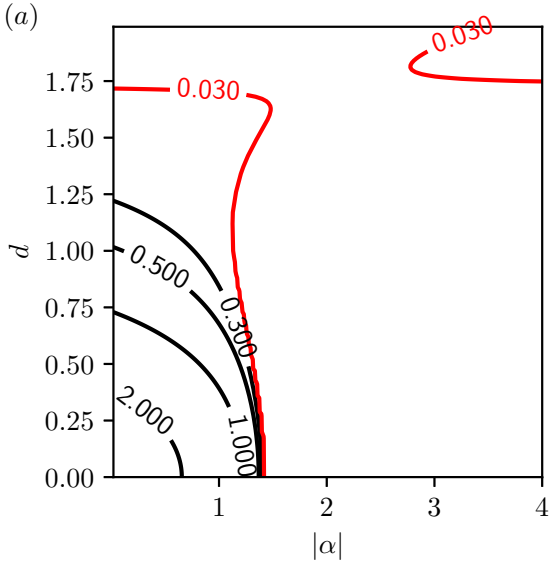

(b)

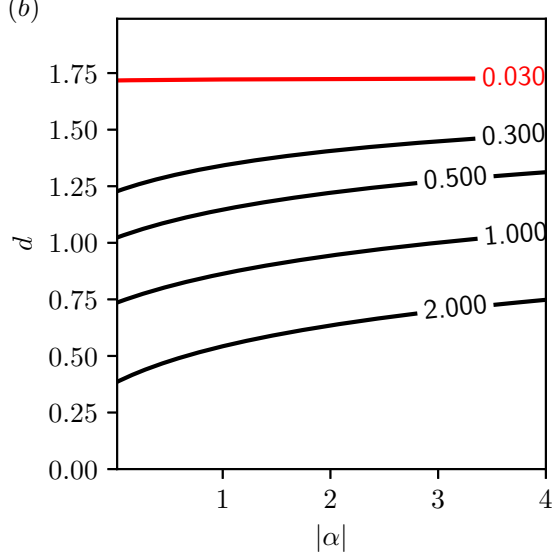

Figure 7. Contours of maximum growth rates of instability for the $2+1$-vortex problem in a three-dimensional, continuously stratified fluid in the place $(|\alpha|, d)$ for $(a) \alpha<0$ and $(b) \alpha>0$.

the equilibrium. It also affects its stability properties. We thus examine the stability properties of the $m+1$-vortex array in the parameter space $(\alpha, d)$ distinguishing the cases $\alpha<0$ and $\alpha>0$ for clarity.

We first present results for $m=2$ in figure 7. The vortex array is unstable for small $d$ and $\alpha>0$. The growth rate of the instability decays as $d$ is increased as a consequence of the decrease of the interaction between the central vortex and the peripheral vortices as seen in figure $7(b)$. The same is true for for $-1.5<\alpha<0$, see figure $7(a)$. A stronger opposite-sign central vortex is however able to stabilise the vortex array. We recover in particular that the $2+1$-vortex array with $\alpha=-2$ is linearly stable as established analytically by Reinaud \& Carton (2015). Results also indicate that the $2+1$-vortex array is unstable for $\alpha \rightarrow 0$, i.e. for a vanishing strength central vortex. In complete absence of a central vortex, a pair of like-sign point vortices, a 2-body system, is stable. The instability observed for the 2+1-vortex, a 3-body system, with $\alpha \rightarrow 0$ is due to the fact that the central vortex lies initially at a hyperbolic critical point (a stagnation point separating two trajectories around the peripheral vortices). Hence, although the peripheral vortices 

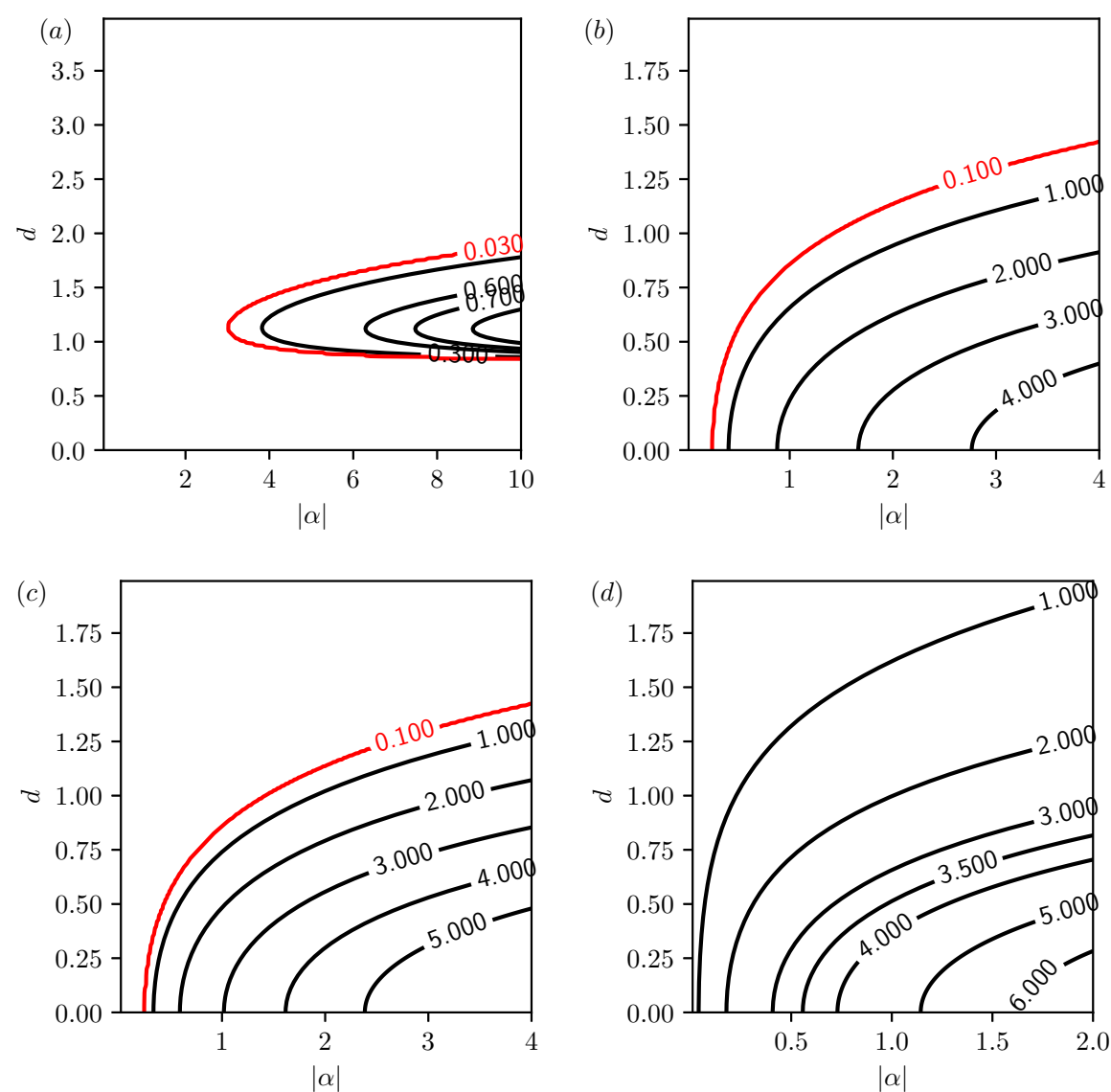

Figure 8. Contours of maximum growth rates of instability for $m$ identical vortices on a ring with an opposite-signed central vortex, $(\alpha<0)$, for QG point vortices in a three-dimensional, continuously stratified fluid in the plane $(|\alpha|, d)$ with $m=3(a), 4(b), 5(c)$ and $6(d)$.

locations are stable, the vanishing central vortex is unstable. This explains the trajectory of the central vortex observed in figure $3(b)$. Similar results are discussed by Kizner $(2014)$ for QG two-layer and single-layer flows, where the pair of active vortices is shown to be stable but an instability can be observed due to the possible motion of the passive central vortex.

Next, results are presented in figure 8 for $\alpha<0$ and in figure 9 for $\alpha>0$ and $3 \leqslant m \leqslant 6$. First, the results show that linearly unstable modes can be found for all values of $m$ investigated, in particular if $|\alpha|$ is larger than a threshold which depends on $m$. Since the influence of the central vortex on the peripheral vortices decreases with its height $d$, this growth rate of the instability is larger for small $d$. This is true for all cases but for $m=3$ and $\alpha<0$, as seen in figure 8. We also confirm that an opposite-signed central vortex favours instability as the threshold in $|\alpha|$ which separates the linearly stable and linearly unstable regions is lower, for a given $d$, when $\alpha<0$. Finally we see that for $\alpha<0$, increasing $m$ makes the vortex array more unstable. On the other hand for $\alpha>0$, we see that increasing $m$ first shifts the threshold in $\alpha$ to larger values. For $m=6$ there is in fact a second, weaker, unstable mode which arises from $(\alpha, d)=(0,0)$ and persists for $\alpha>0$ and $d \neq 0$, see figure $10(a)$. Recall indeed that the array of 6 point vortex is 

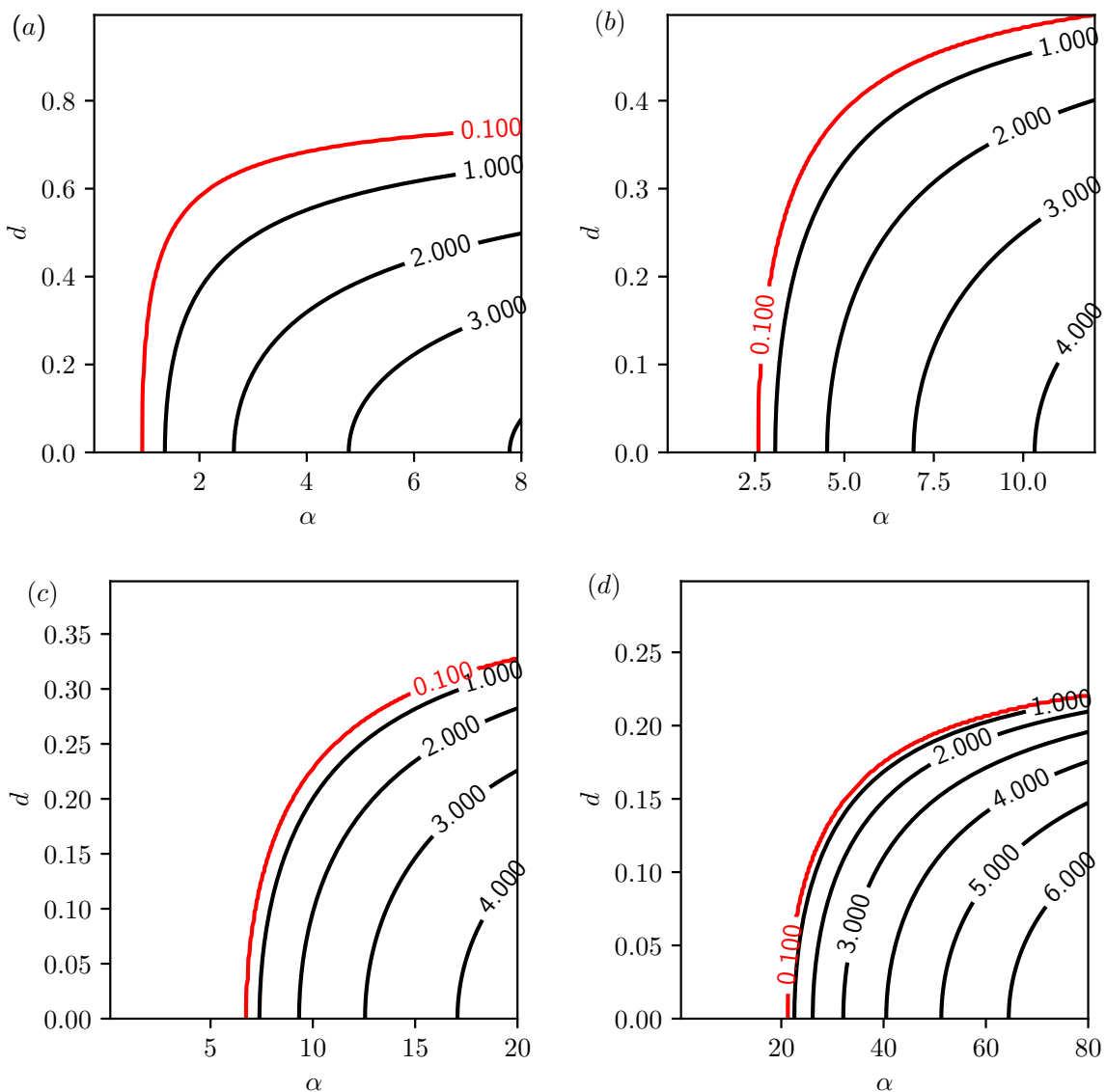

Figure 9. Contours of maximum growth rates for $n$ identical vortices on a ring with an like-signed central vortex for QG point vortices in a three-dimensional, continuously stratified fluid in the place $(|\alpha|, d)$ for $m=3(a), 4(b), 5(c)$ and $6(d)$. The panel for $m=6$ offers a close-up for small $d$. Results of $m=6$ and larger $d$ are shown in figure 10 .

unstable. Therefore, one expects for $m \geqslant 6$ that when $\alpha \rightarrow 0$, the $m+1$-vortex problem to be linearly unstable as well. This mode becomes the dominant mode for $m \geqslant 7$, see figure 10. The particular case $m=6$ is interesting as in that case we see that a weak central vortex located in the plane of the ring is able to stabilise the vortex array, while instability is recovered if the weak central vortex is vertically offset by a value of $d$ of order of the ring radius $R=1$.

\section{Finite volume vortices}

We next turn our attention to finite volume vortices of uniform potential vorticity. The objective of this part is to provide the generic shapes and characteristics of finite core equilibria for $2 \leqslant m \leqslant 7$ at highest possible resolution. We again investigate vortex arrays with and without a central vortex. We do not intend to provide at this stage a complete catalogue of the equilibria throughout a large parameter space, which arguably would be of little interest. There is an additional length scale in the problem, associated with the size of the vortices. In this problem, the radius of the ring along which the peripheral vortices lie is no longer fixed but is varied. Recall that this distance sets the distance 

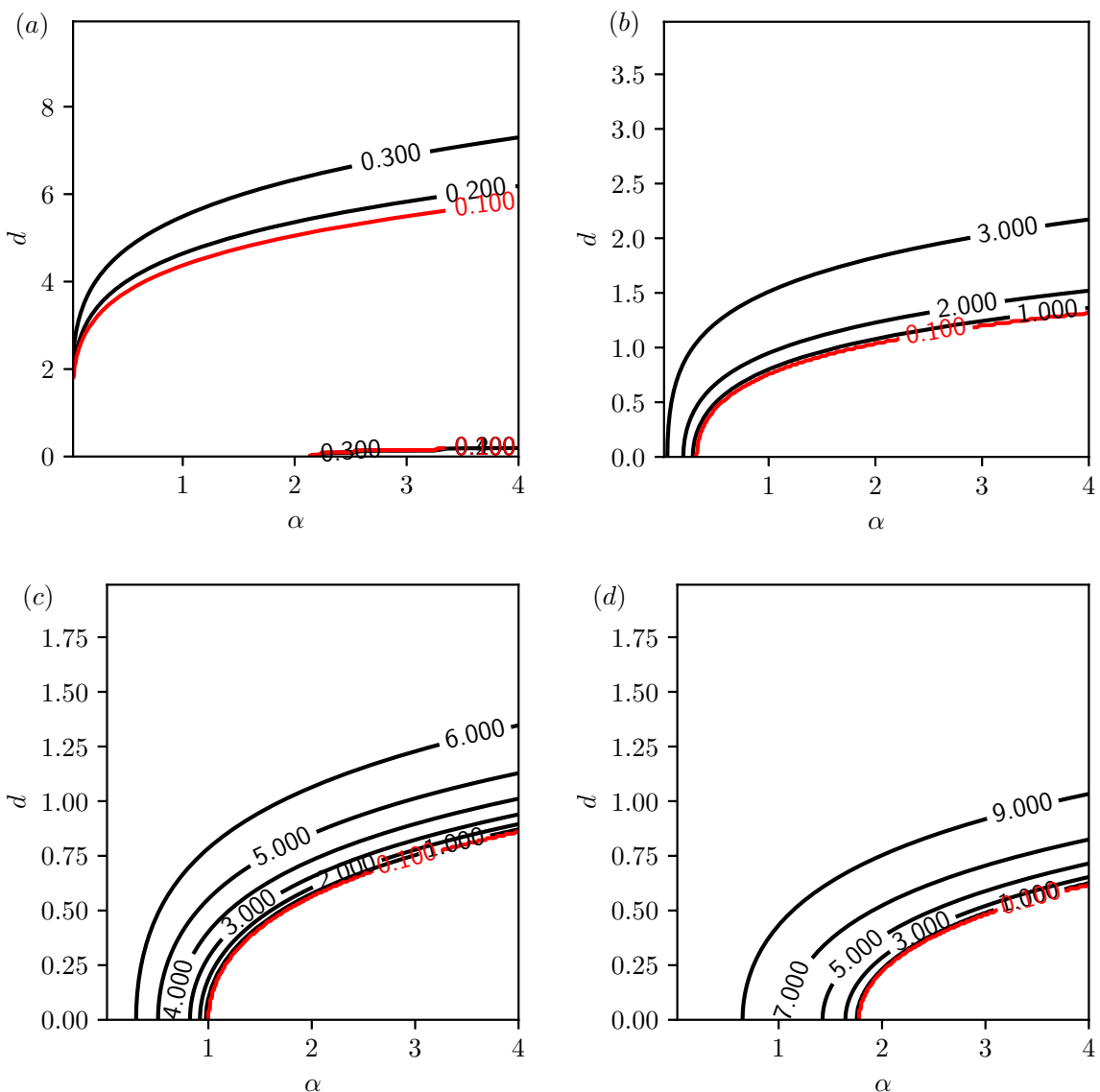

Figure 10. Contours of maximum growth rates for $n$ identical vortices on a ring with an like-signed central vortex for QG point vortices in a three-dimensional, continuously stratified fluid in the place $(|\alpha|, d)$ for $m=6(a), 7(b), 8(c)$ and $9(d)$.

between the vortices. We are still free to fix a length scale in the problem. Without loss of generality we set the height of the vortices to 1 , in the reference frame vertically stretched by $N / f$. We restrict attention to vortex having a unit mean height-to-width aspect ratio, $h_{v} / r_{v}$, measured in the stretched reference frame due to the numerical cost of the computations. Here, $h_{v}$ is the half height of the vortices and $r_{v}$ is their mean horizontal radius. Such vortices have therefore a unit Burger number $B u=\left(h_{v} / r_{v}\right)^{2}=1$. The vortices are however pancake-like in physical space. The specific choice of unit heightto-width aspect ratio vortices is however consistent with findings in QG turbulence were it has been shown that vortices have typically a near unit aspect ratio (close to 0.8 ), see Reinaud, Dritschel \& Koudella (2003). It should be noted that this specific choice imposes limitation on the variety of equilibria we investigate.

The branches of equilibria stem from vortex arrays of infinitely distant spherical vortices in the vertically stretched reference frame. The vortices volume is $V=4 \pi h_{v} r_{v}^{2} / 3=$ $\pi / 6$, and their $\mathrm{PV}$ is set to $q=4 \pi$. This means that the time scale associated with the vortices, inversely proportional to $q$, and their strength $q V$ is the same in all experiments. The $m$ vortices are each discretised in the vertical direction by $n_{c}=83$ horizontal layers. The vortex bounding contours are discretised by $n_{p}=4 n_{c}$ nodes. It should be noted that 
the numerical cost of the algorithms grows as $m^{2} n_{p}^{2} n_{c}^{2}$. We focus on equilibria having imposed symmetries.

First, all $m$ vortices have the same shape. Vortex $i, 2 \leqslant i \leqslant m$, is the image of vortex 1 by a rotation of angle $2(i-1) \pi / m$. Second, each vortex is symmetric with respect to a vertical plane passing through the vortex centre and the vertical axis of rotation (the $z$-axis). Similar numerical setups and symmetries are imposed for the $m+1$-vortex problem. We focus on cases where the central vortex lies in the plane containing the ring of peripheral vortices $(d=0)$. We consider the two cases where the PV of the central vortex is $q_{0}= \pm 4 \pi$. In these cases, the central vortex has an imposed $m$-fold symmetry, and the number of nodes $n_{p}$ discretising each contour is adjusted to be divisible by $m$. Even when reducing the computational load by taking advantages of the problem symmetries, determining a single equilibrium state at these resolutions for $7+1=8$ vortices can take up to a day on a single core of a modern processor. This imposes limitations on the number of states one can determine.

For each configuration, and a given value of $m$, we determine the family of equilibrium states spanned by the ratio of a distance $\delta$ separating the peripheral vortices and the centre of the domain, which is the centre of rotation, to the mean vortex radius $r_{v}=0.5$. Branches of solutions are sought until we reach an ending point of the solution branch, namely when the vortices touch. The choice of the distance to be used depends on the configuration as not all distances between a point defined on the vortices and the rotation centre varies monotonously along the solution branches, due to the vortices deformation. In practice, the distance $\delta$ is the distance between the innermost edge of the vortices and the centre of the ring when the peripheral vortices develop a sharp inner edge forms. On the other hand, $\delta$ is the distance between the outermost edge and the centre of the ring when the vortices develop a flat, or slightly convex inner surface. We start the branches of solution from large separation distances. When an equilibrium is reached by the iterative method, the distance is reduced and the numerical method is resumed for the new separation. It should be noted that if the radius of the ring tends to infinity, spherical vortices should be in mutual equilibrium. The shape of the vortices departs from a sphere as the vortices are located closer together. The equilibrium vortices are deformed to be able to steadily withstand the shear (proportional to $r^{-3}$ ) they induce on each other. The numerical method used to determine the equilibrium states is iterative and is described in Appendix B.

We determine the equilibria for the $m$-vortex problem for $2 \leqslant m \leqslant 7$. It should be noted that the equilibria for $m=2$ were originally obtained at lower resolution by Reinaud \& Dritschel (2002) and at high resolution in Reinaud \& Dritschel (2018a).

We first describe the shape of the equilibria obtained numerically and we address next their linear stability. The method used for the linear stability analysis is described in Appendix C. Figure 11 shows a top view on the vortex bounding contours at the end of the solution branch, where the vortices nearly touch for $m=2$ to $m=7$. Except for $m=2$, the vortices near the ending point of the branch do not exhibit a single sharp inner edge. This is in contrast with the two-dimensional equilibria shown by Dritschel (1985) (in particular their figure 2) where a single sharp inner edge forms for $m \leqslant 4$. Instead, for $m \geqslant 3$ the innermost part of the vortices flattens and remains slightly convex. Vortices touch by their side at the ending point of the solution branch.

Figure 12 shows the maximum growth rate $\sigma^{\max }=\max \left\{\sigma_{r}\right\}$ versus the distance $\delta$ between the innermost edge of the vortices and the centre of rotation. Results show that the equilibria are unstable for $\delta$ less than a threshold depending on $m$. Importantly, unstable equilibria are found for all $m$ for all three configurations. Finite volume vortices can deform and therefore can be sensitive to deformation modes as well as displacement 

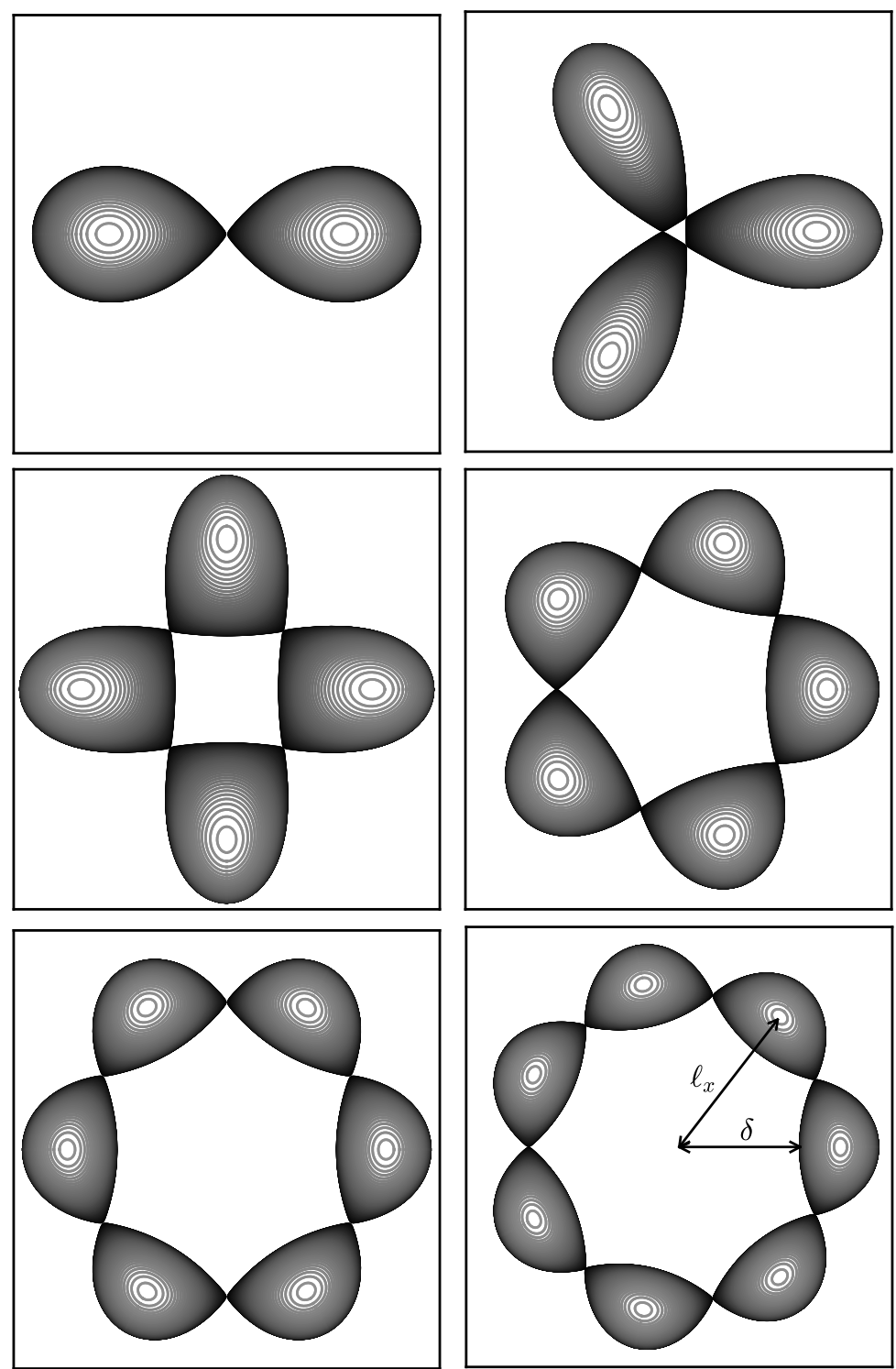

FiguRE 11. Top view on the vortex bounding contours to the $m$-vortex equilibria at the end of the solution branch where the vortices touch. The grey scale indicates the height of the contour: lighter grey contours are nearer the top.

modes. By construction, only the displacement modes can be captured by the analysis of the stability of systems of point vortices. Moreover, there is a fundamental difference between deformation modes which affect the shape of the vortices and displacement modes which move the vortices. If a point vortex equilibrium is sensitive to a displacement mode the point vortex equilibrium is fundamentally unstable, i.e. it is unstable for all separation distances between the vortices. The distance influences the magnitude of the growth rate, but does not change the nature of the stability properties. On the other hand, the deformation modes for finite volume vortices can be triggered when the vortices are close enough to each other, as it is seen in figure 12. In practice such deformation modes are associated with the phase-locking of vorticity Rossby waves travelling on the 


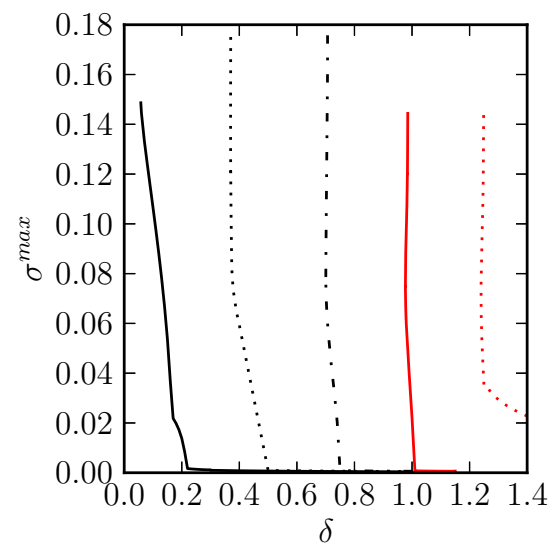

FIGURE 12. Maximum growth rate $\sigma^{\max }$ of instability vs the inner gap $\delta$ for the finite volume $m$-vortex problem for $m=3$ (solid black), 4 (dotted black), 5 (dashed-dotted black), 6 (solid red), and 7 (dotted red).

vortex boundaries, see Dritschel (1995) for a discussion for two-dimensional vortices. A mode with non-zero growth rate is observed for $m=7$ for all distances and is associated with the displacement mode. The growth rate of the instability increases as the vortices are closer together. This is related to the increase of the strain vortices induced on each other. It should however be noted that the similar (but much weaker) displacement instability mode for $m=6$ observed in the point vortex calculation is not clearly noticeable for the finite volume vortex equilibria. We conjecture that the deformed finite volume vortices have adapted to the external shear induced by the other vortices. This has weakened the already weak mode, and its growth rate cannot be convincingly distinguished from background numerical noise. Indeed the equilibria are obtained numerically by an iterative method, described in the appendix B. The iterative method is stopped when the correction to the rotation rate of the vortex array is less than a threshold $\left(10^{-11}\right.$ in the present study). This means that a small residual unsteadiness remains together with the unavoidable small truncation errors inherent to the numerical approach. Hence very weak instabilities may have a growth rate of the order of the precision of our numerical calculation of the equilibrium.

Figure 13 shows the vortex equilibria for the $m+1$-vortex problem when $d=0$ and $\alpha=-1$. Recall that, except for $m=3$, the associated point vortex arrays are unstable. The general shape of the equilibria is qualitatively similar to the shape of the twodimensional equilibria obtained by Kizner et al. (2007) and the vortex arrays obtained experimentally by Trieling et al. (2010). It should be noted that we have not been able to reach an ending point in the case $m=2$ despite numerous attempts. Importantly, the algorithm requires that we fix a distance between two points discretising the vortices during the iterative procedure to determine the equilibrium. When the equilibrium is reached, this distance is reduced and the procedure is resumed for this new distance. As mentioned previously not all distances vary monotonously along the branch of solutions, and we may need to describe the solution branch piece by piece, adapting the adequate distance to be fixed. In the case $m=2$ we were not able to find a convenient distance to be fixed that would allow one to go further along the branch. A new approach may be required in this case.

The presence of an opposite-signed vortex in the centre of the structure changes the topology of the streamlines hence the shape of the equilibria. This difference with the 

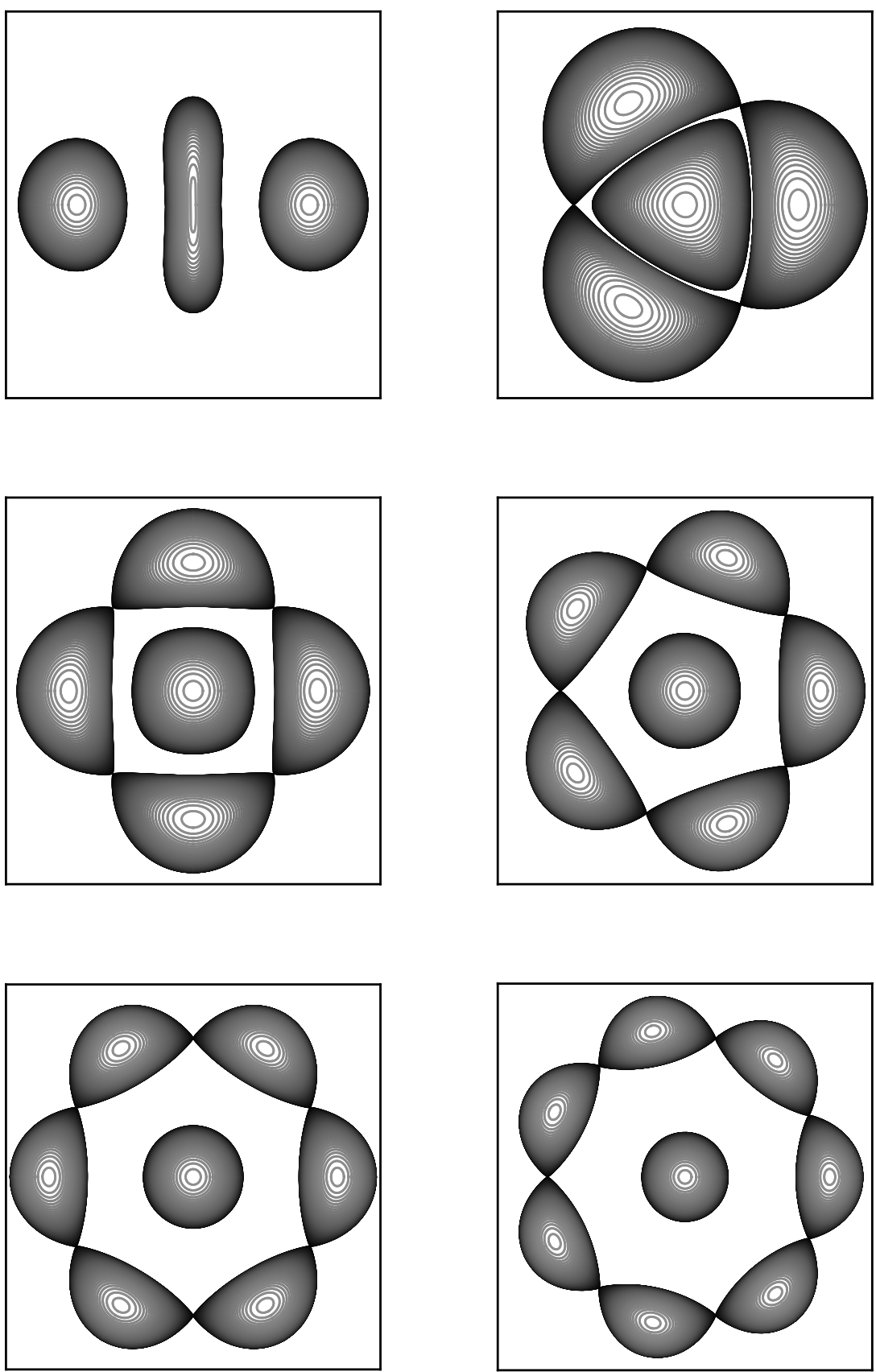

FiguRE 13. Top view on the vortex bounding contours to the $m+1$-vortex equilibria at the end of the solution branch where the vortices touch (except for $m=2$ in the top left panel where this state could not be obtained numerically). The central vortex and the peripheral vortices have opposite signed PV. The grey scale indicates the height of the contour: lighter grey contours are nearer the top. 


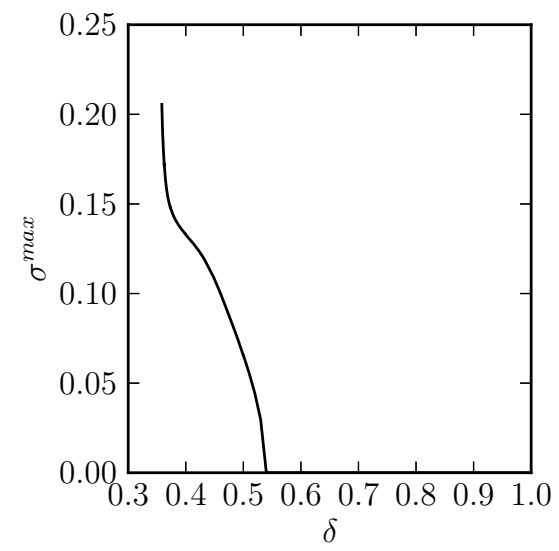

FIGURE 14. Maximum growth rate $\sigma^{\max }$ of instability vs the inner gap $\delta$ for the finite volume $m+1$-vortex problem for $m=3$ where the central vortex and the peripheral vortices have opposite sign.

$m$-vortex problem becomes less noticeable for large $m$ as the vortices remain far from the central vortex even at the ending point of the branch. However, the peripheral vortices tend to be thinner in the radial direction when the opposite-signed central vortex is present. It is however important to stress that these qualitatively similar vortex shapes have fundamentally different stability properties.

Figure 14 shows the maximum growth rate $\sigma^{\max }$ for the $3+1$ vortex problem with $d=0$ and $\alpha=-1$. We do not show results for the other values of $m$ as they are all unstable for all distances. Indeed these configurations are all unstable to at least displacement modes as found for the point vortices. The linear stability of the finite volume vortices confirmed this. The case $m=3$ is the only one which is linearly stable to displacement modes. Again, we see that deformation modes can destabilise the equilibrium if the vortices are close enough together. Recall vortices at equilibrium are more deformed as they are closer together.

Finally, figure 15 illustrates the equilibria at the ending point of the solution branches for the $m+1$-vortex problem with $d=0$ and $\alpha=1$. Due to the presence of the central likesigned vortex, the vortex exhibit a sharp inner edge for $m \leqslant 6$. Note that for $m>6$, the distance between two neighbouring peripheral vortices is less than the distance between these vortices and the central vortex. In these cases, the peripheral vortices interact more strongly with their neighbours on the ring. This explains the change in the shape of the equilibria.

The maximum growth rate $\sigma^{\max }$ of the unstable modes versus $\delta$ are shown in figure 16. Again, unstable modes are obtained for $\delta$ less than a threshold for all $m$. The weak unstable mode observed for the $3+1$ point vortex system for $d=0$ and $\alpha=1$ is not clearly captured for the finite volume problem. We conjecture that, as seen for the 6vortex problem, the deformation of the vortices, hence their capacity to adapt to the external shear weakens the instability. The growth rate of the instability is of order of the background numerical noise.

For the sake of completeness one can also calculate two of the fundamental invariants of the equilibria, namely their total energy $E$ and their angular impulse $J$ defined by

$$
E=-\frac{1}{2} \iiint_{V} q \psi \mathrm{d} v
$$



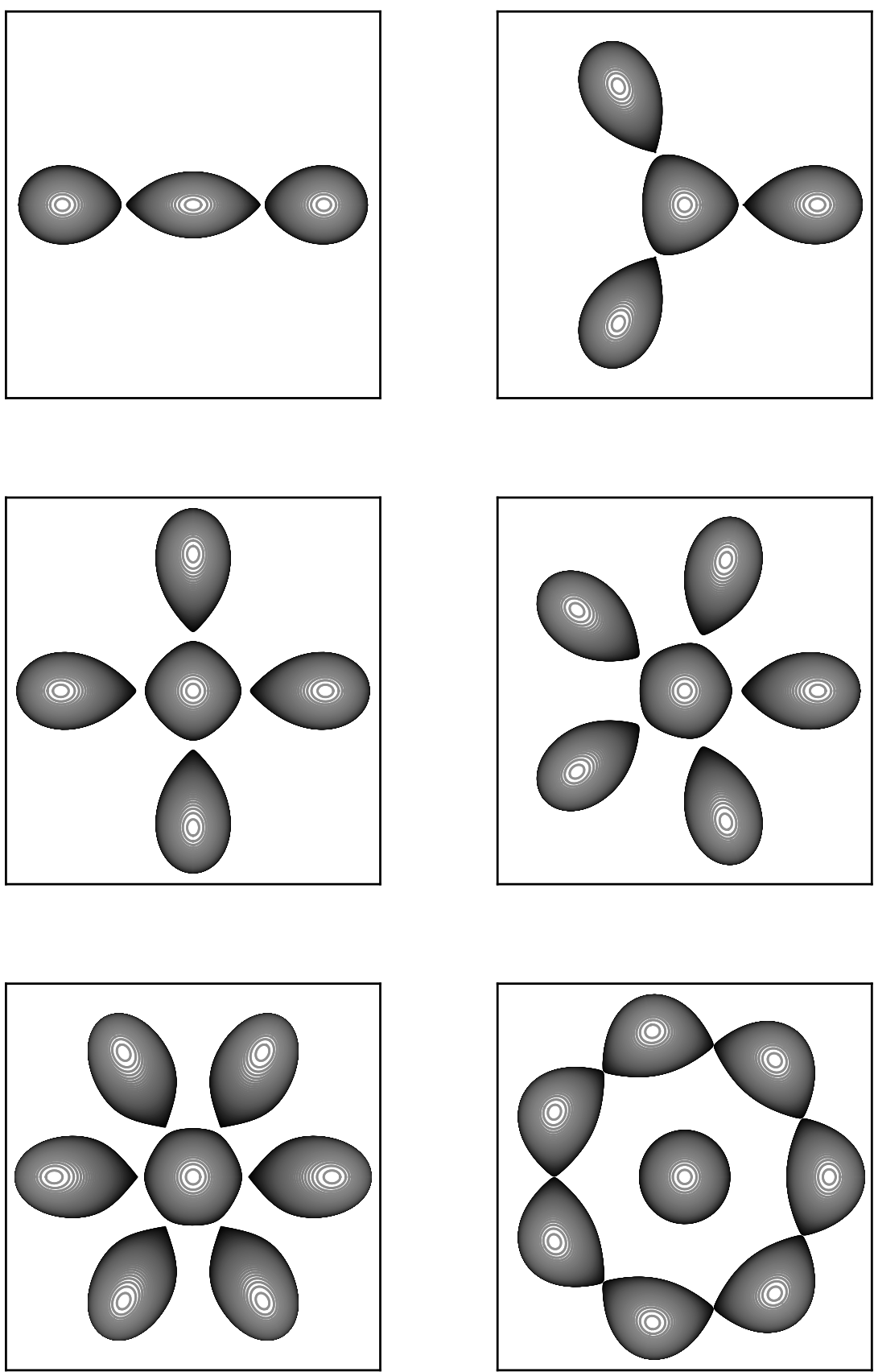

FiguRE 15. Top view on the vortex bounding contours to the $m+1$-vortex equilibria at the end of the solution branch where the vortices touch. The central vortex and the peripheral vortices have same PV. The grey scale indicates the height of the contour: lighter grey contours are nearer the top. 


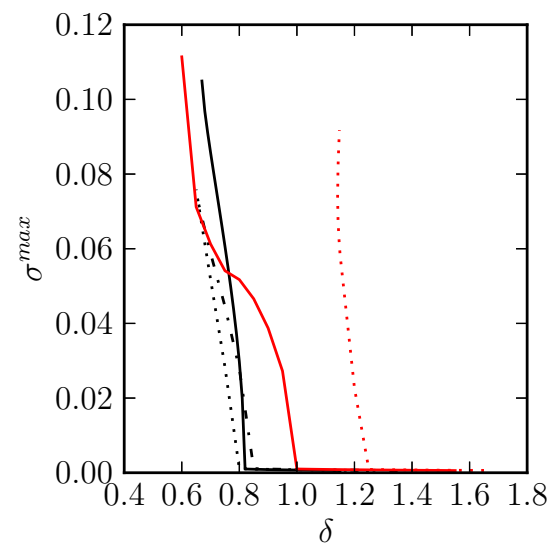

FIGURE 16. Maximum growth rate $\sigma^{\max }$ of instability vs the inner gap $\delta$ for the finite volume $m+1$-vortex problem where the central vortex and the peripheral vortices have same sign for $m=3$ (solid black), 4 (dotted black), 5 (dashed-dotted black), 6 (solid red) and 7 (dotted red).

$$
J=\frac{1}{2} \iiint_{V} q\left(x^{2}+y^{2}\right) \mathrm{d} v .
$$

A summary of the results are presented in figure 17. For the two-vortex problem, the margin of stability has been observed empirically to coincide with the maximum of $E$ and minimum of $J$ versus a distance separating the vortices, see Reinaud \& Dritschel (2002) and Reinaud \& Dritschel (2005). The fact that the combined extrema can be associated with the onset of instability has been justified by Saffman (1992) using Kelvin's variational principle. The condition is a sufficient but not necessary condition. We have not found a systematic match between the onset of instability and the coincidence of extrema for $E$ and $J$ for $m>2$. It should be noted that such a match should not be expected at least for the displacement mode as its origin is independent of the distance separating the vortices. For almost all cases, the rotation rate $\omega$ and the energy $E$ increase as the vortices are closer to one another hence their interaction is stronger. On the other hand, $J$ decreases with the distance as $J$ is associated with a volume integral of PV weighted by distance to the system centre squared. Only for the case $m=3$ and $d=0, \alpha=-1$ these trends differ. In this case the peripheral vortices are close to the highly-deformed opposite-signed central vortex. This central vortex dominates the overall rotation (which becomes clockwise) and its contribution to the angular impulse is negative.

We next illustrate the nonlinear evolution of unstable equilibria for the three categories of vortex arrays. We start with an example of the 3-vortex problem. The equilibria corresponds to a state with an innermost gap $\delta=0.16$ and the maximum growth rate of instability is $\sigma^{\max }=0.037$. Results obtained with the Lagrangian Contour Dynamics method are presented in figure 18. The equivalent equilibrium with point vortices is linearly stable indicating that there is no unstable displacement mode. The instability is associated with the deformation of the vortices. We do not force the instability but simply let it grow from pseudo-random numerical errors. For numerical efficiency the number of nodes discretising the contours of the equilibrium $\left(n_{p}=4 n_{c}\right)$ is reduced while maintaining high accuracy. This is done by 'renoding' the contours which is part of Contour Surgery with standard setup parameters. This procedure is enough to introduce a small perturbation on the vortex shape. Figure 18 shows the vortices in the 

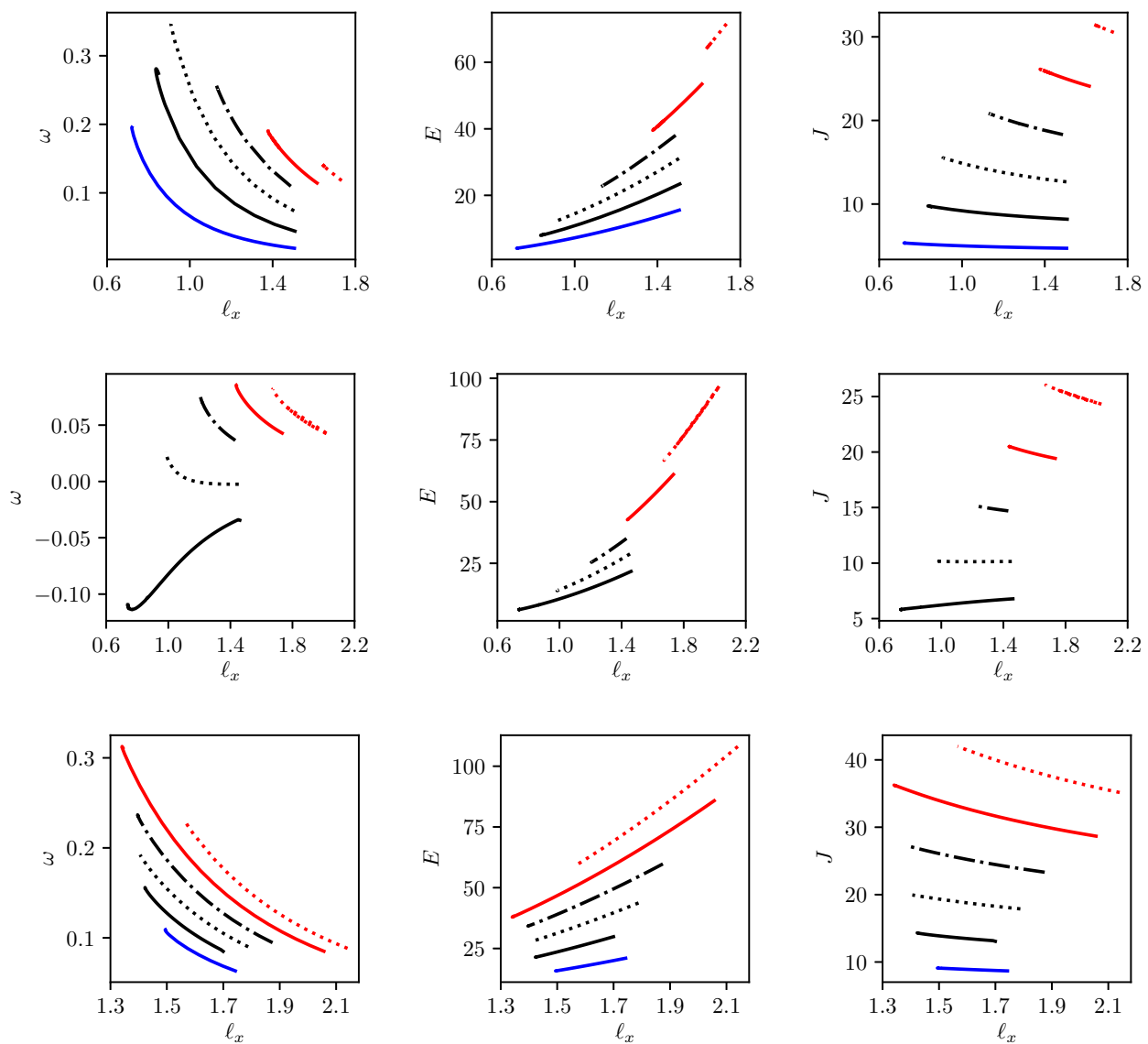

FIGURE 17. Global diagnostics for the equilibrium state: rotation rate $\omega$, total energy $E$, and angular impulse $J$ versus $\ell_{x}$ the distance between the centroid of the peripheral vortices and the centre of the ring for $m=2$ (solid blue), $m=3$ (solid black), $m=4$ (dotted black), $m=5$ (dashed-dotted black), $m=6$ (solid red), $m=7$ (dotted red) and for the $m$-vortex problem (top row), $m+1$-vortex problem with $d=0, \alpha=-1$ (middle row) and the $m+1$-vortex problem with $d=0$ and $\alpha=1$ (bottom row).

reference frame steadily rotating with the equilibrium. Therefore any motion observed in this reference frame represents a departure from equilibrium. The vortices remain at equilibrium for a long period of time, while the deformation slowly grows from the low numerical noise. By $t=22$, the inner most edges of the vortices have deformed and have formed bridges which connect them to the neighbouring vortex to their right (in the direction of rotation of the structure). This deformation is qualitatively similar to the one associated with the merger of a pair of like-signed vortices, see Reinaud \& Dritschel (2002). As the flow develops, the merged vortex forms a complex structure which resembles a three-blade propeller. Some PV from the central layers converges to the centre of the structure. To conserve the angular impulse $J$, some PV from the lower and upper layers of the vortices is ejected away from the centre of the structure. These will turn into filaments and small scale debris in the late evolution of the flow.

The second illustration of the nonlinear evolution of the equilibria concerns an unstable $6+1$ vortex equilibrium with $d=0$ and $\alpha=-1$. Recall that such a vortex array is sensitive to a displacement mode. Results obtained with the Lagrangian Contour 

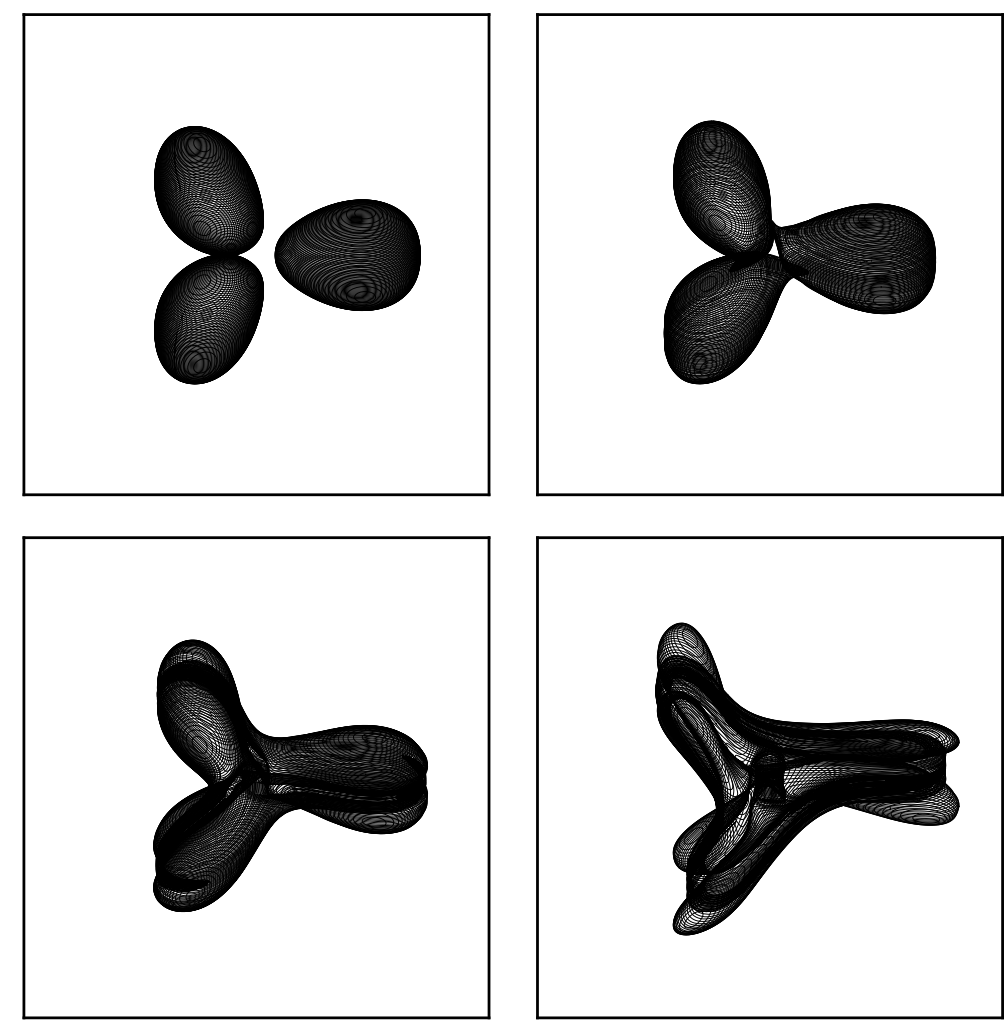

FiguRE 18. Evolution of the vortex bounding contours for an unstable $\left(\sigma^{\max }=0.037\right)$ array of three vortices in mutual equilibrium for $\delta=0.16$ at $t=0,22,24$ and 27.5. The vortex bounding contours are viewed orthographically at an angle of $45^{\circ}$ from the vertical.

Dynamics method are shown in figure 19. Here, the distance $\delta$ between the peripheral vortex innermost edge and the centre of the central vortex is $\delta=1.08$ and the most unstable mode has a growth rate $\sigma^{\max }=0.12$. We first observe that the vortices indeed move. Some vortices getting closer together creating a larger gap with their other neighbouring vortex on the ring. The straining field changes as the vortices depart from their equilibrium. The vortices thus start to deform. The vortices which have got closer together merge to form larger structures. These structures are not stable and can further interact and/or break back into much deformed secondary structures. It resembles instances of partial merger observed during the interaction between two vortices, see Reinaud \& Dritschel (2002).

Finally, we consider an example of unstable $4+1$ vortex equilibrium with $d=0$ and $\alpha=1$ with $\delta=0.16$ and $\sigma^{\max }=0.076$. The equivalent point vortex equilibrium is linearly stable. Results obtained with the Lagrangian Contour Dynamics method are presented in figure 20. In this case, the instability is associated with the deformation of the vortices. The sharp inner edge of the peripheral vortices elongates and a filament of $\mathrm{PV}$ is shed near the edge of at least one vortex. This filament is wrapped around the central vortex. This in turn breaks the symmetry of the flow. As the vortices deform, some of the peripheral vortices strongly interact with the central vortex in an asymmetric way. This is followed by a series of partial mergers, and breaking into secondary vortices. In the latter two cases the central vortex remains near the centre of the domain at least 

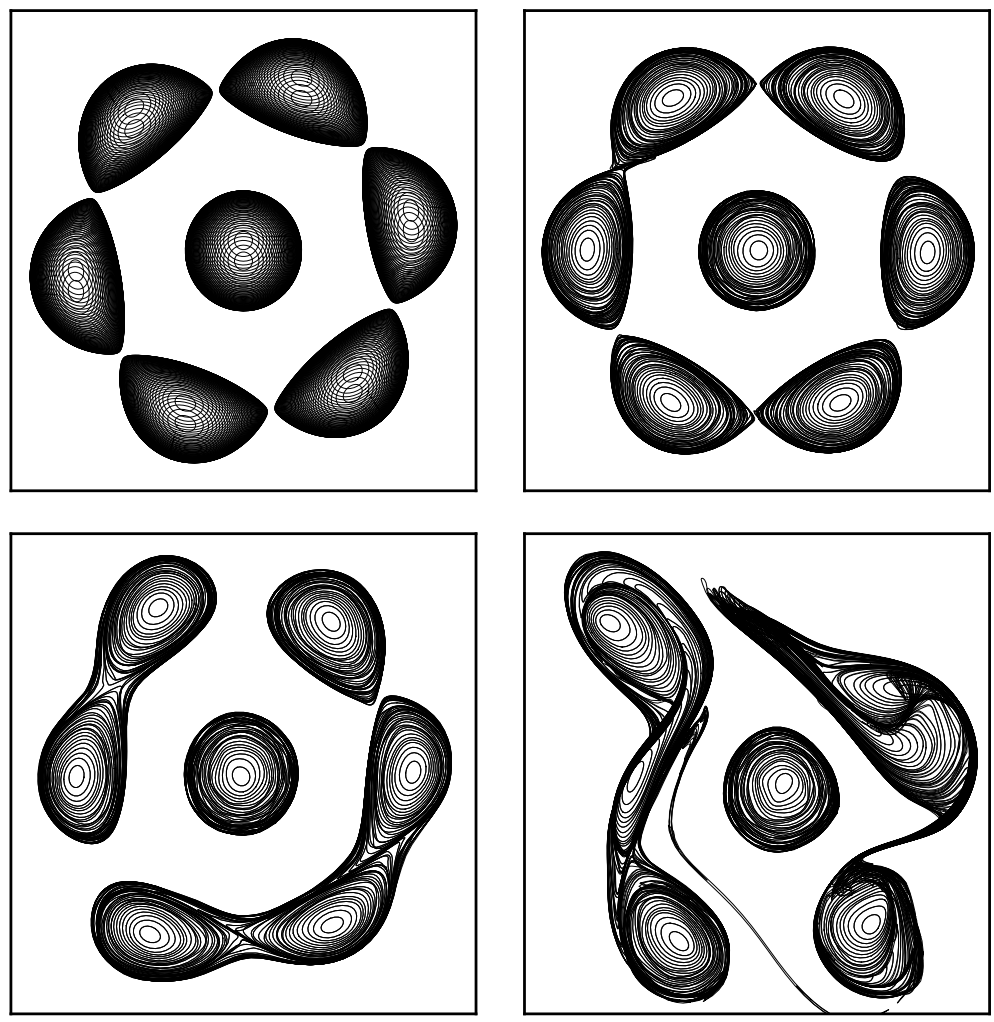

Figure 19. Evolution of the vortex bounding contours for an unstable $\left(\sigma^{\max }=0.12\right)$ array of six peripheral vortices and an opposite-signed central vortex $(\alpha=-1)$ with $d=0$, in mutual equilibrium for $\delta=1.08$ at $t=0,12,16$ and 21. The vortex bounding contours are viewed from the top.

for the duration of the simulation. This is due to conservation of the linear and angular impulses, as overall the system must remain anchored to the centre of the domain.

\section{Conclusion}

We have investigated the problem of $m$ - and $m+1$-vortices in mutual equilibrium in the context of three-dimensional, unit Burger number, quasi-geostrophic vortices. We have first shown that these vortices have specific stability properties even if they exhibit overall similar patterns with their known two-dimensional counterparts. Notably, in the absence of a central vortex, no more than 5 identical three-dimensional quasi-geostrophic vortices can remain stably located on a circular ring. Recall that 7 two-dimensional vortices can remain in mutual equilibrium for long times. We have also seen that adding an opposite signed vortex on the vertical axis of rotation of the system generally tends to destabilise the vortex array. The opposite trend is observed if the central vortex has the same sign as the peripheral ones, except for $m=2$. However, instability can be found in general if the central vortex is strong enough. Moreover, finite volume vortices can be sensitive to deformation modes when the vortices are close enough to each other. Nonetheless, there exists large parts of the parameter space where such vortex arrays are stable and therefore can persist in time. The existence of such equilibria can explain the formation of patterns in geophysical contexts and on the atmosphere of other planets such as the 

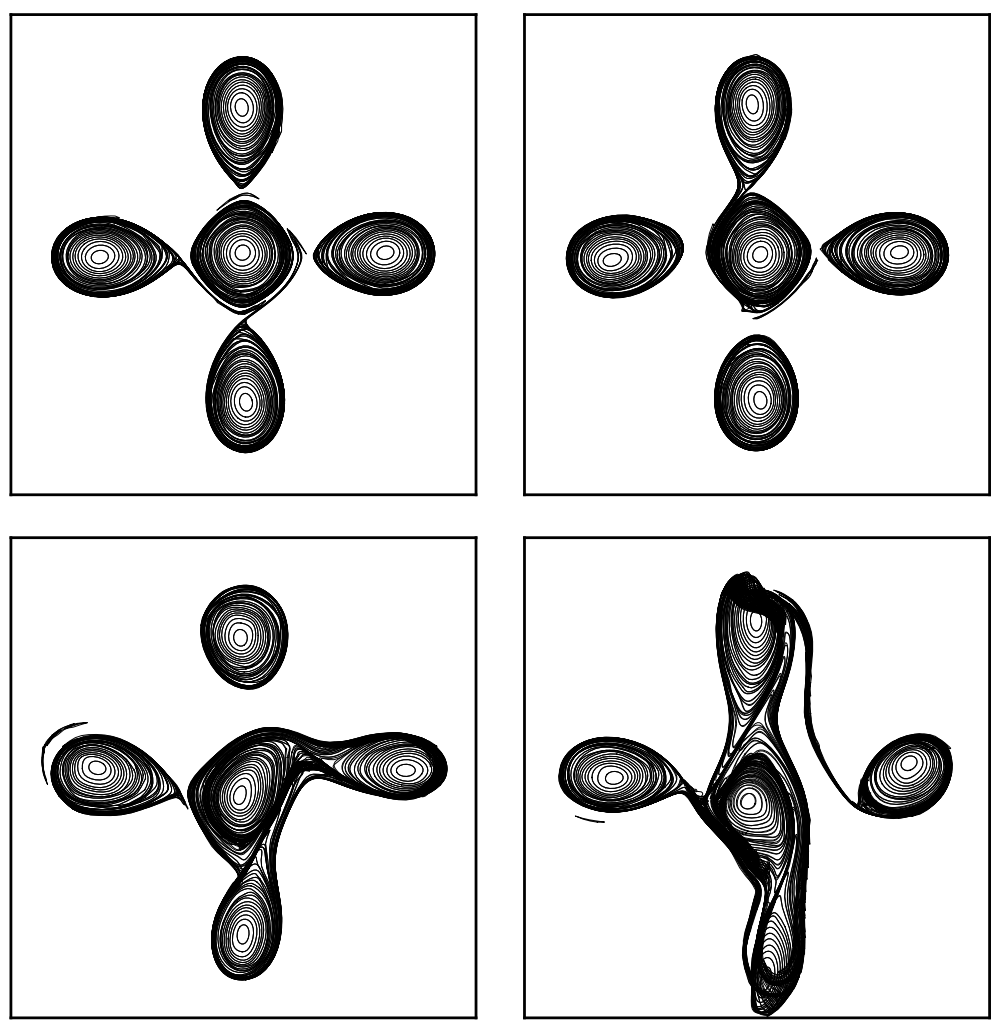

FiguRE 20. Evolution of the vortex bounding contours for an unstable $\left(\sigma^{\max }=0.076\right)$ array of four peripheral vortices and an like-signed central vortex $(\alpha=1)$ with $d=0$, in mutual equilibrium for $\delta=0.16$ at $t=10,14,20$ and 24 . The vortex bounding contours are viewed from the top.

polygonal clusters of cyclones observed in the polar regions of the Jovian atmosphere, see Adriani et al. (2018). The paper has focused for the first time on these equilibria on the simplest three-dimensional context relevant to environments subject to rapid background rotation and stable density stratification. This research can be extended to further studies in other contexts with different vertical density stratification in particular when the Boussinesq approximation fails to be relevant. Additionally, the present investigation has restricted attention to unit Burger number vortices, which limits the variety of equilibria investigated. Due to the numerical cost of computing three-dimensional finite volume equilibria and addressing their stability, it is impractical to perform an exhaustive study of the influence of the Burger number in this context. Such a study can however be performed using a two-layer model and will be considered in a future work.

\section{Appendix A. Linear stability for point vortices}

This appendix briefly describes the method used to address the linear stability of a system of point vortices. It relies on a straightforward linearisation of the equations of motions of the vortices. The $m$ peripheral vortices of strength $\kappa_{i}$ are located on a ring of radius $R$ at a polar angle $\left\{\theta_{i}\right\}_{1 \leqslant i \leqslant m}$,

$$
\theta_{i}=\frac{i-1}{m} 2 \pi
$$


The peripheral vortices have a strength $\kappa_{i}=\kappa$ for $1 \leqslant i \leqslant m$. The central vortex is located at $(0,0, d)$ and has strength $\kappa_{0}$.

Vortex 1 is located at the point $(R, 0,0)$ and is used to evaluate the uniform rotation angular velocity $\omega$

$$
\omega=\frac{v_{1}}{R}=\frac{\kappa_{0}}{\left(R^{2}+d^{2}\right)^{3 / 2}}+\kappa \sum_{i=2}^{m} \frac{1-\cos \theta_{i}}{\left.R^{3}\left(\left(1-\cos \theta_{i}\right)\right)^{2}+\sin ^{2} \theta_{i}\right)^{3 / 2}},
$$

where $v_{1}$ is the velocity of vortex 1 in the $y$-direction.

It should be noted that equation (A 2) can be simplified but the simplified formula is not particularly illuminating. We also denote for simplicity the Cartesian coordinates of the point vortices $\left(x_{i}, y_{i}, z_{i}\right)$, and we define

$$
r_{i j}^{2}=\left(x_{i}-x_{j}\right)^{2}+\left(y_{i}-y_{j}\right)^{2}+\left(z_{i}-z_{j}\right)^{2} .
$$

We next focus on the normal (exponentially growing) modes of perturbation. We consider perturbations of the horizontal coordinates of the vortices

$$
\left(\tilde{x}_{i}, \tilde{y}_{i}, 0\right)=e^{\sigma t}\left(x_{i}^{\prime}, y_{i}^{\prime}, 0\right) .
$$

where $\sigma=\sigma_{r}+\mathrm{i} \sigma_{i} \in \mathbb{C}$. The real part $\sigma_{r}$ of $\sigma$ corresponds to the growth rate of the mode while its imaginary part $\sigma_{i}$ is its frequency. We do not consider vertical perturbations, which would otherwise also affect the distance separating the vortices, since the vertical advection is negligible in the QG model, hence no perturbation stemming from an external quasi-geostrophic flow may move the vortices in the vertical direction. We do not consider perturbations of the strength of the vortices either. It should be noted that the vortex strengths are materially conserved in the incompressible, inviscid and adiabatic flow. Other works, in particular Carnevale \& Kloosterziel (1994) have however included such perturbations, and they may lead to instability. We do not consider algebraic modes nor nonlinear modes in this work.

The equations for the perturbations are obtained by linearising the equations of motion of the vortices in the reference frame rotating with the vortices about their equilibrium position:

$$
\begin{gathered}
\frac{\mathrm{d} x_{i}^{\prime}}{\mathrm{d} t}=\sigma x_{i}^{\prime}=\omega y_{i}^{\prime}- \\
\sum_{j=0, j \neq i}^{m} \frac{\kappa_{j}}{r_{i j}^{3}}\left[\left(1-3 \frac{\left(y_{i}-y_{j}\right)^{2}}{r_{i j}^{2}}\right)\left(y_{i}^{\prime}-y_{j}^{\prime}\right)-3 \frac{\left(y_{i}-y_{j}\right)\left(x_{i}-x_{j}\right)}{r_{i j}^{2}}\left(x_{i}^{\prime}-x_{j}^{\prime}\right)\right] \\
\frac{\mathrm{d} y_{i}^{\prime}}{\mathrm{d} t}=-\sigma y_{i}^{\prime}=-\omega x_{i}^{\prime}+ \\
\sum_{j=0, j \neq i}^{m} \frac{\kappa_{j}}{r_{i j}^{3}}\left[\left(1-3 \frac{\left(x_{i}-x_{j}\right)^{2}}{r_{i j}^{2}}\right)\left(x_{i}^{\prime}-x_{j}^{\prime}\right)-3 \frac{\left(y_{i}-y_{j}\right)\left(x_{i}-x_{j}\right)}{r_{i j}^{2}}\left(y_{i}^{\prime}-y_{j}^{\prime}\right)\right]
\end{gathered}
$$

This provides a $2 m \times 2 m$ for the $m$-vortex problem $((2 m+2) \times(2 m+2)$ for the $m+1$ vortex problem respectively) algebraic eigenvalue problem for $\sigma_{j}, 1 \leqslant j \leqslant 2 m(2 m+2$, respectively) which is solved numerically. 


\section{Appendix B. Finding finite-size equilibria}

The numerical method to obtain steadily rotating states is an iterative method which makes the vortex bounding contours in each horizontal cross-section converge to streamlines, $\psi=$ constant, in the relevant rotating reference frame. In this reference frame, the velocity is tangent to the contours, and the contours do not deform in time. Here, each vortex is represented by a collection of $n_{c}=83$ horizontal contours $\mathcal{C}_{k}$.

The streamfunction in the rotating frame $\tilde{\psi}_{k, n}$ along the $k^{\text {th }}$ contour $\left(\mathcal{C}_{k}\right)$ in the $n^{\text {th }}$ iteration takes the form:

$$
\tilde{\psi}_{k, n}\left(\boldsymbol{\rho}_{k, n}\right)=\psi_{k, n}\left(\boldsymbol{\rho}_{k, n}\right)-\frac{1}{2} \Omega_{n} \boldsymbol{\rho}_{k, n}^{2}
$$

where $\boldsymbol{\rho}_{k, n}=\left(x_{k, n}, y_{k, n}\right)$ is the horizontal position vector describing the contour $\mathcal{C}_{k}, \Omega_{n}$ is the $n^{t h}$ estimate for the background rotation, and $\psi_{k, n}\left(\boldsymbol{\rho}_{k, n}\right)$ is the streamfunction obtained from the inversion of Poisson's equation (2.1).

For the $n+1^{s t}$ iteration, we enforce, approximately, the condition of equilibrium, namely:

$$
\tilde{\psi}_{k, n+1}\left(\boldsymbol{\rho}_{k, n+1}\right)=c_{k}
$$

where $c_{k}$ is a constant (generally different for each contour $\mathcal{C}_{k}$ ). Starting from the $n^{\text {th }}$ guess for the equilibriam solution, this equation is partially linearised about $\boldsymbol{\rho}_{k, n}$ to find the correction $\boldsymbol{\rho}_{k, n+1}-\boldsymbol{\rho}_{k, n}$. We use here radial corrections $\eta_{k, n}$, where the radius is measured horizontally from the centroid of the vortex to which $\mathcal{C}_{k}$ belongs. We write

$$
\boldsymbol{\rho}_{k, n+1}=\boldsymbol{\rho}_{k, n}+\eta_{k, n} \hat{\boldsymbol{r}}_{k, n}
$$

where $\hat{\boldsymbol{r}}_{k, n}$ is the unit vector in the radial direction. Equation (B 2) is then expanded to first order in $\eta$, but ignoring the implicit change in $\psi$ associated with the change in the contour shapes:

$$
\begin{aligned}
\tilde{\psi}_{k, n+1}\left(\boldsymbol{\rho}_{k, n+1}\right) \simeq & \psi_{k, n}\left(\boldsymbol{\rho}_{k, n}\right)-\frac{1}{2} \Omega_{n} \boldsymbol{\rho}_{k, n}^{2}-\frac{1}{2} \Omega^{\prime} \boldsymbol{\rho}_{k, n}^{2} \\
& +\eta_{k, n}\left[\left(\hat{\boldsymbol{r}}_{k, n} \cdot \boldsymbol{\nabla}\right) \psi_{k, n}\left(\boldsymbol{\rho}_{k, n}\right)-\Omega_{n} \boldsymbol{\rho}_{k, n} \cdot \boldsymbol{r}_{k, n}\right]
\end{aligned}
$$

where

$$
\Omega^{\prime}=\Omega_{n+1}-\Omega_{n}
$$

is the correction to the background rotation rate and

$$
\psi_{k, n+1} \simeq \psi_{k, n}
$$

is heuristically assumed, following Pierrehumbert (1980). The latter assumption avoids the large matrix problem that would otherwise result.

Thus, from equations (B 2) and (B 4), the correction $\eta_{k, n}$ can be expressed as a simple function of the set of constants $c_{k}$ and $\Omega^{\prime}$ :

$$
\eta_{k, n}=\frac{c_{k}-\tilde{\psi}_{k, n}\left(\boldsymbol{\rho}_{k, n}\right)+\frac{1}{2} \Omega^{\prime} \boldsymbol{\rho}_{k, n}^{2}}{\left(\hat{\boldsymbol{r}}_{k, n} \cdot \boldsymbol{\nabla}\right) \psi_{k, n}\left(\boldsymbol{\rho}_{k, n}\right)-\Omega_{n} \boldsymbol{\rho}_{k, n} \cdot \hat{\boldsymbol{r}}_{k, n}}
$$

Two other conditions are next imposed to determine the rotation rate correction $\Omega^{\prime}$ 
and the constants $c_{k}$. The first one is the volume conservation of the vortices. In the absence of vertical mass transfer in QG flows, volume conservation is equivalent to the conservation of the area $\mathcal{A}_{k}$ within each contour $\mathcal{C}_{k}$. This allows one to write a first relation between $c_{k}$ and $\Omega^{\prime}$. Area conservation between two iterations can be expressed, at the first order, by

$$
\begin{aligned}
A_{k, n+1} & =\frac{1}{2} \oint_{\mathcal{C}_{k}} \boldsymbol{\rho}_{k, n+1}^{2} \mathrm{~d} \theta \\
& \simeq A_{k, n}+\oint_{\mathcal{C}_{k}} \eta_{k, n} \hat{\boldsymbol{r}}_{k, n} \cdot \boldsymbol{\rho}_{k, n} \mathrm{~d} \theta \equiv \mathcal{A}_{k}
\end{aligned}
$$

where $\theta$ is the geometric polar angle, $A_{k, n}$ is the area of the $k^{t h}$ contour at the $n^{t h}$ iteration, and $\mathcal{A}_{k}$ is the xprescribed area of $\mathcal{C}_{k}$ (note $A_{k, n}$ converges to $\mathcal{A}_{k}$ as $\eta_{k, n} \rightarrow 0$ ). Substituting $\eta_{k, n}$ from (B 7) into equation (B 8), we obtain the following equations for the constants $c_{k}$ as a function of $\Omega^{\prime}$ :

where (with $w_{k, n} \equiv \xi_{k, n}^{-1} \boldsymbol{r}_{k, n} \cdot \boldsymbol{\rho}_{k, n}$ )

$$
c_{k}=h_{k, n}-\Omega^{\prime} g_{k, n}
$$

$$
h_{k, n}=\frac{\mathcal{A}_{k}-A_{k, n}+\oint_{\mathcal{C}_{k}} \tilde{\psi}_{k, n} w_{k, n} \mathrm{~d} \theta}{\oint_{\mathcal{C}_{k}} w_{k, n} \mathrm{~d} \theta} \text { and } g_{k, n}=\frac{1}{2} \frac{\oint_{\mathcal{C}_{k}} \rho_{k, n}^{2} w_{k, n} \mathrm{~d} \theta}{\oint_{\mathcal{C}_{k}} w_{k, n} \mathrm{~d} \theta} \text {. }
$$

A second constraint is next imposed to determine the rotation rate correction $\Omega^{\prime}$. We fix the distance between one point chosen on one of the vortices of the ring. This implies that the local correction at this point is zero:

$$
\eta_{k_{1}, n}\left(\theta_{1}\right)=0
$$

Here $k_{1}$ refers to the mid-contour of the chosen vortex and the point has with polar angle $\theta_{1}$. Using equation (B 7), we find

$$
\Omega^{\prime}=\frac{\tilde{\psi}_{k_{1}, n}\left(\theta_{1}\right)-h_{k_{1}, n}}{(1 / 2) \boldsymbol{\rho}_{k_{1}, n}^{2}\left(\theta_{1}\right)-g_{k_{1}, n}}
$$

The specific choice for the point depends on the geometry of the equilibrium. In practice it is either the innermost or the outermost edges of the contour in the plane $z=0$ of the vortex whose centre lies on the semi-axis $[0, \infty)$.

The iterative scheme is repeated until the correction $\Omega^{\prime}$ is less than a given tolerance, namely $10^{-11}$ in the present study.

\section{Appendix C. Linear stability for finite-size vortices}

We present here the method used to address the linear stability of the finite-size vortices. We consider infinitesimal disturbances of the horizontal position vector $\boldsymbol{\rho}_{k}$ of points along the contour $\mathcal{C}_{k}$ from its equilibrium value $\boldsymbol{\rho}_{e, k}=\left(x_{e}, y_{e}\right)$,

$$
\boldsymbol{\rho}_{k}(\tilde{\theta}, t)=\boldsymbol{\rho}_{e, k}+\gamma_{k} \frac{\left(-\mathrm{d} y_{e} / \mathrm{d} \tilde{\theta}, \mathrm{d} x_{e} / \mathrm{d} \tilde{\theta}\right)}{\left(\mathrm{d} x_{e} / \mathrm{d} \tilde{\theta}\right)^{2}+\left(\mathrm{d} y_{e} / \mathrm{d} \tilde{\theta}\right)^{2}}
$$

where $\gamma_{k}$ is a disturbance area taken in the form 


$$
\gamma_{k}(\theta, t)=\mathrm{e}^{\sigma t} \sum_{m}^{M} \hat{\gamma}_{k, m} \mathrm{e}^{\mathrm{i} m \theta} .
$$

Here, the angle $\tilde{\theta}$ is the 'travel-time coordinate', an angle proportional to the time taken by a fluid particle to travel along the contour $\mathcal{C}_{k}$. The mode $m=1$ corresponds to a displacement of the contour. The evolution of the disturbance area $\gamma_{k}$ is governed at first order by

$$
\frac{\partial \gamma_{k}}{\partial t}+\omega_{k} \frac{\partial \gamma_{k}}{\partial \tilde{\theta}}=-\sum_{l=1}^{N} \Delta q_{l} \frac{\partial}{\partial \tilde{\theta}} \oint_{\mathcal{C}_{l}} \gamma_{l} G_{k, l}(\rho) \mathrm{d} \tilde{\theta}^{\prime}
$$

where $N=n_{v} \times n_{c}$ is the total number of contours, $\omega_{k}$ is the constant rotation rate of the fluid particle along contour $\mathcal{C}_{k}, \Delta q_{l}$ is the PV jump across contour $\mathcal{C}_{l}, G_{k, l}$ is the Green's function giving the velocity induced in the layer containing $\mathcal{C}_{k}$ by the PV in the layer containing contour $\mathcal{C}_{l}$ in an unbounded infinite domain and $\rho=\left|\boldsymbol{\rho}_{e, k}(\theta)-\boldsymbol{\rho}_{e, l}\left(\theta^{\prime}\right)\right|$. Substituting $\gamma_{k}$ and $\gamma_{l}$ by their expression from equation (C 2) into equation (C 3) leads to a $2 \times N \times M$ real eigenvalue problem for the real and imaginary parts of $\gamma_{k}$. The eigenvalues $\sigma$ are complex. Their real part $\sigma_{r}$ are the mode growth rates and their imaginary parts $\sigma_{i}$ are the mode frequencies. The eigenvectors $\left\{\tilde{\gamma}_{n}\right\}_{1 \leqslant n \leqslant 2 N M}$ allow one to reconstruct the spatial structure of the modes. We use $M=10$.

It should be noted that the perturbation considered include all possible deformation of the vortex boundary (including the displacement of the vortex), consistent with the QG equations: although the perturbation can have any vertical structure, no vertical displacement of the layers is allowed.

\section{REFERENCES}

Adriani, A., Mura, A., Orton, G., Hansen, C., Altieri, F., Moriconi, M. L., Rogers, J., Eichstdt, G., Momary, T., Ingersoll, A. P., Filacchione, G., Sindoni, G., Tabataba-Vakili, F., Dinelli, B. M., Fabiano, F., Bolton, S. J., Connerney, J. E. P., Atreya, S. K., Lunine, J. I., Tosi, F., Migliorini, A., Grassi, D., Piccioni, G., Noschese, R., Cicchetti, A., Plainaki, C., Olivieri, A., Oneill, M. E., Turrini, D., Stefani, S., Sordini, R. \& Amoroso, M. 2018 Clusters of cyclones encircling Jupiter's poles. Nature 555, 216-219.

Aref, H. 2009 Stability of relative equilibria of three vortices. Phys. Fluids 21, 094101.

BurbeA, J. 1982 On patches of uniform vorticity in a plane of irrotational flow. Arch. Ration. Mech, Anal. 77, 349-358.

Carnevale, G. F. \& Kloosterziel, R. .C. 1994 Emergence and evolution of triangular vortices. J. Fluid Mech. 259, 305-331.

Chelton, D. B., Schlax, M. G. \& Samelson, R. M. 2011 Global observations of nonlinear mesoscale eddies. Prog. Oceanogr. 91, 161-216.

Crowdy, D. G. 2002 Exact solutions for rotating vortex arrays with finite-area cores. J. Fluid Mech. 469, 209-235.

Crowdy, D. G. 2003 Polygonal n-vortex arrays: A Stuart model. Phys. Fluids 15 (12), 37103717.

Dijkstra, H.A. 2008 Dynamical oceanography. Berlin Heidelberg: Springer-Verlag.

Dritschel, D. G. 1985 The stability and energetics of corotating uniform vortices. J. Fluid Mech. 157, 95-134.

Dritschel, D. G. 1988 Contour surgery: A topological reconnection scheme for extended integrations using contour dynamics. J. Comput. Phys. 77, 240-266.

Dritschel, D. G. 1995 A general theory for two-dimensional vortex interactions. J. Fluid Mech. 293, 269-303. 
Dritschel, D. G. 2002 Vortex merger in rotating stratified flows. J. Fluid Mech. 455, 83-101.

Dritschel, D. G. \& SARAVAnAN, R. 1994 Three-dimensional quasi-geostrophic contour dynamics, with an application to stratospheric vortex dynamics. Quart. J. Roy. Meteorol. Soc. 120, 1267-1297.

Ebbesmeyer, C. C., Taft, B. A., McWilliams, J. C., Shen, C. Y., Riser, S. C., Rossby, H. T., Biscaye, P. E. \& Östlund, H. .G. 1986 Detection, structure and origin of extreme anomalies in a western Atlantic oceanographic section. J. Phys. Ocean. 16, 591612.

Gryanik, V. M. 1983 Dynamics of localized vortex perturbations on vortex charges in a baroclinic fluid. Izv. Atmos. Acean. phy. 19, 347-352.

Kizner, Z. 2011 Stability of point-vortex multipoles revisited. Phys. Fluids 23, 064104.

Kizner, Z. 2014 On the stability of two-layer geostrophic point-vortex multipoles. Phys. Fluids 26, 046602.

Kizner, Z. \& Khvoles, R. 2004a The tripole vortex: Experimental evidence and explicit solutions. Phys. Rev. E $\mathbf{7 0}(1), 016307$.

Kizner, Z. \& Khvoles, R. 2004b Two variations on the theme of lambchaplygin: Supersmooth dipole and rotating multipoles. Regular Chaotic Dyn. 9, 509.

Kizner, Z., Khvoles, R. \& McWilliams, J. C. 2007 Rotating multipoles on the $f$ - and $\gamma$-planes. Phys. Fluids 19 (1), 016603.

Kizner, Z., Shteinbuch-Fridman, B., Makarov, V. \& Rabinovich, M. 2017 Cycloidal meandering of a mesoscale eddy. Phys. Fluids 29, 086601.

Kurakin, L. G. \& Yudovich, V. I. 2002 The stability of stationary rotation of a regular vortex polygon. Chaos 12 (3), 574-595.

Morikawa, G. K. \& Swenson, E. V. 1971 Interacting motion of rectilinear geostrophic vortices. Phys. Fluids 14 (6), 10581073.

Peterson, M. .R., Williams, S. J., Maltrud, M. E., Hecht, M. W. \& Hamann, B. 2013 A three-dimensional eddy census of a high-resolution global ocean simulation. J. Geophys. Res. Oceans 118, 1757-1774.

Pierrehumbert, R. T. 1980 A family of steady, translating vortex pairs with distributed vorticity. J. Fluid Mech. 99, 129-144.

Reinaud, J. N. \& CARTON, X. 2015 Existence, stability and formation of baroclinic tripoles in quasi-geostrophic flows. J. Fluid Mech. 785, 1-30.

Reinaud, J. N. \& Carton, X. 2016 The interaction between two oppositely travelling, horizontally offset, antisymmetric quasi-geostrophic hetons. J. Fluid Mech. pp. 409-443.

Reinaud, J. N. \& Dritschel, D. G. 2002 The merger of vertically offset quasi-geostrophic vortices. J. Fluid Mech. 469, 297-315.

Reinaud, J. N. \& Dritschel, D. G. 2005 The critical merger distance between two co-rotating quasi-geostrophic vortices. J. Fluid Mech. 522, 357-381.

Reinaud, J. N. \& Dritschel, D. G. 2009 Destructive interactions between two counterrotating quasi-geostrophic vortices. J. Fluid Mech. 639, 195-211.

Reinaud, J. N. \& Dritschel, D. G. 2018a The merger of geophysical vortices at finite Rossby and Froude number. J. Fluid Mech. 848, 388-410.

Reinaud, J. N. \& Dritschel, D. G. $2018 b$ The stability and nonlinear evolution of quasigeostrophic toroidal vortices. J. Fluid Mech. under review.

Reinaud, J. N., Dritschel, D. G. \& Koudella, C. R. 2003 The shape of vortices in quasigeostrophic turbulence. J. Fluid Mech. 474, 175-192.

Reinaud, J. N., Sokolovskiy, M. A. \& Carton, X. 2017 Geostrophic tripolar vortices in a two-layer fluid: Linear stability and nonlinear evolution of equilibria. Phys. Fluids 29 (3), 036601.

Safman, P. G. 1992 Vortex Dynamics. Cambridge, UK: Cambridge University Press.

Shteinbuch-Fridman, B., Makarov, V. \& Kizner, Z. 2015 Two-layer geostrophic tripoles comprised by patches of uniform potential vorticity. Phys. Fluids 27, 036602.

Shteinbuch-Fridman, B., Makarov, V. \& Kizner, Z. 2017 Transitions and oscillatory regimes in two-layer geostrophic hetons and tripoles. J. Fluid Mech. 810, 535-553.

Sokolovskiy, M. A. \& Verron, J. 2008 On the motion of $a+1$ vortices in a two-layer rotating fluid. In IUTAM Symposium on Hamiltonian Dynamics, Vortex Structures, Turbulence, IUTAM Bookseries Vol. 6, p. 481. Springer. 
Thomson, J. J. 1883 A treatise of vortex rings. London: MacMillan and Co.

Trieling, R. R., van Heijst, G. J. F. \& Kizner, Z. 2010 Laboratory experiments on multipolar vortices in a rotating fluid. Phys. Fluids 22, 094104.

VAlLIS, G. K. 2006 Atmospheric and oceanic fluid dynamics: fundamentals and large-scale circulation. Cambridge, UK: Cambridge University Press.

Wu, H. M., Overman II, E. A. \& Zabusky, N. J. 1984 Steady-state solutions of the Euler equations: rotating and translating v-states with limiting cases. part i. numerical algorithms and results. J. Comput. Phys. 53 (1), 4271.

Xue, J. J., Johnson, E. .R. \& McDonald, N. R. 2017 New families of vortex patch equilibria for the two-dimensional Euler equations. Phys. Fluids 29 (12), 123602.

ZhANG, Z., WANG, W. \& QIU, B. 2014 Oceanic mass transport by mesoscale eddies. Science 345, 322-324. 